Calibration and correction procedure for quantitative out-of-plane shearography

This content has been downloaded from IOPscience. Please scroll down to see the full text.

2015 Meas. Sci. Technol. 26045201

(http://iopscience.iop.org/0957-0233/26/4/045201)

View the table of contents for this issue, or go to the journal homepage for more

Download details:

IP Address: 157.193.240.1

This content was downloaded on 11/06/2015 at $14: 54$

Please note that terms and conditions apply. 


\title{
Calibration and correction procedure for quantitative out-of-plane shearography
}

\author{
Filip Zastavnik ${ }^{1}$, Lincy Pyl ${ }^{1}$, Jun $\mathrm{Gu}^{1}{ }^{1}$, Hugo Sol ${ }^{1}$, Mathias Kersemans ${ }^{2}$ and \\ Wim Van Paepegem ${ }^{2}$ \\ ${ }^{1}$ Department of Mechanics of materials and construction, Vrije Universiteit Brussel (VUB), Pleinlaan 2, \\ 1050 Brussels, Belgium \\ ${ }^{2}$ Department of Materials science and engineering, Universiteit Gent, Technologiepark-Zwijnaarde 903, \\ 9052 Zwijnaarde, Belgium
}

E-mail: filip.zastavnik@vub.ac.be

Received 25 September 2014, revised 5 November 2014

Accepted for publication 24 November 2014

Published 20 February 2015

\begin{abstract}
Quantitative shearography applications continue to gain practical importance. However, a study of the errors inherent in shearography measurements, related to calibration of the instrument and correction of the results, is most often lacking. This paper proposes a calibration and correction procedure for the out-of-plane shearography with a Michelson interferometer. The calibration is based on the shearography measurement of known rigidbody rotations of a flat plate and accounts for the local variability of the shearing distance. The correction procedure further compensates for the variability of the sensitivity vector and separates the two out-of-plane deformation gradients when they are coupled in the measurement. The correction procedure utilizes two shearography measurements of the same experiment with distinct shearing distances. The effectiveness of the proposed calibration procedure is demonstrated in the case of a static deformation of a centrally loaded plate, where the discrepancy between experimental and finite element analysis results is minimized.
\end{abstract}

Keywords: shearography, correction, calibration, shearing distance, sensitivity vector

(Some figures may appear in colour only in the online journal)

\section{Introduction}

Shearography is an optical full-field measurement method $[1,2]$. It uses interference of coherent laser light to produce images that correspond to the displacement gradient of the observed surface. Most commonly, shearography setups are sensitive to the out-of-plane displacement gradients, but configurations sensitive to the in-plane displacement gradients also exist. The strength of shearography lies in its ability to visualize very small displacement gradients, on the $\mu \mathrm{m} \mathrm{m}^{-1}$ level, on relatively large surfaces (order of $\mathrm{cm}^{2}$ to $\mathrm{m}^{2}$ ). The size of the surface area that can be successfully measured depends on the power of the laser light source.

Since its introduction [3, 4], shearography has predominantly been used qualitatively, as a non-destructive testing (NDT) technique $[5,6]$. In composite industry it is used to detect material faults, such as laminate debonding [7]; in the pneumatic tire manufacturing it is used to detect defects in tires [8]. Qualitative fault detection is often used by heatloading the specimens and visualizing the heterogeneous deformation around the defect, with an example shown in figure 1(a). Fault detection can be semi-automated and enhanced numerically to estimate the size and depth of the defect [9], but it still relies on the skill of the operator to assess the damage.

Quantitative applications of shearography are less developed. The focus of most quantitative work in the literature is on strain measurement [10]. The order of magnitude of the strain measurements makes it suitable for the quantification of the residual stress [11]. The experimental setup of shearography can be modified, such that instead of measuring the deformation of a surface, it measures the shape of a surface [12]. Another application of quantitative shearography is the full-field stiffness identification using displacement gradients of vibrating surfaces. An example of local stiffness identification of vibrating beams $[13,14]$ is shown in figure $1(b)$. 


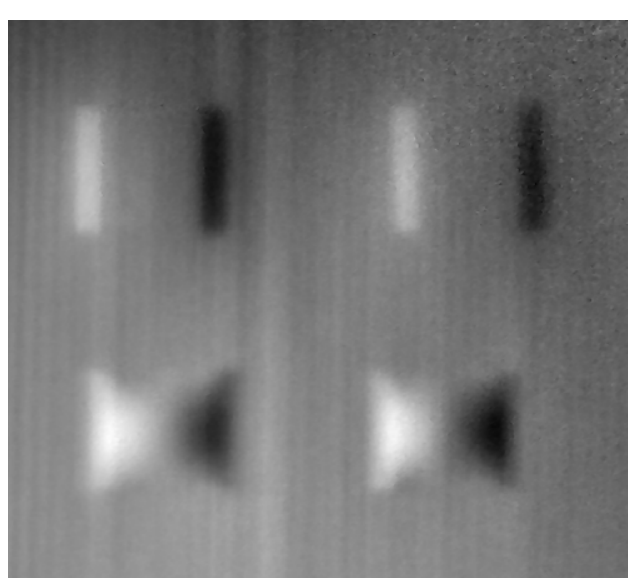

(a)
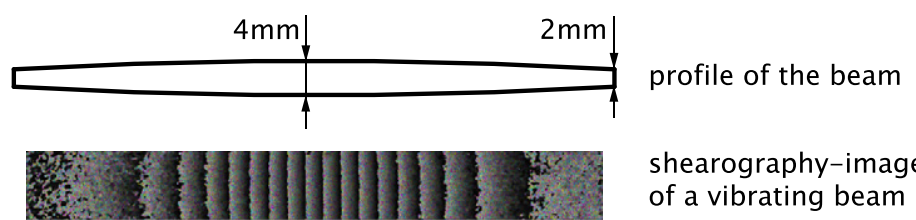

shearography-image of a vibrating beam

Stiffness El $\left[\mathrm{Nm}^{2}\right]$

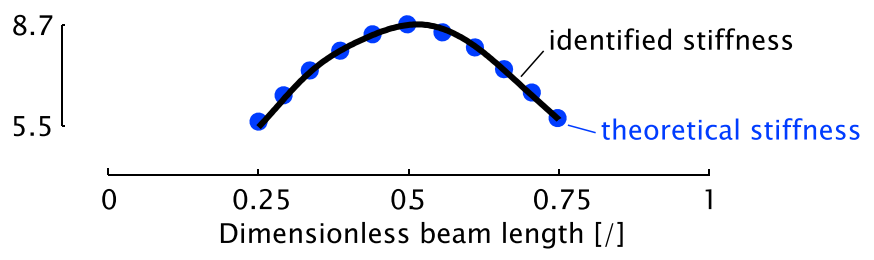

(b)

Figure 1. An example of qualitative shearography is delamination detection-four intentional subsurface delaminations in a composite plate are revealed by thermally loading the surface and visualizing it with shearography $(a)$. A quantitative example is the identification of the local bending stiffness $E I$ of a vibrating aluminium beam with variable cross-section [13] $(b)$.

Shearography is sensitive to the displacements and deformations in the order of magnitude of the wavelength of light, thus very accurate measurements should be possible. However, it is also susceptible to high levels of noise and systematic error sources (discussed further in the paper) which make obtaining reliable measurements difficult. The ability to accurately calibrate a setup and have an estimate of the uncertainty of the result would benefit the quantitative application of shearography. The standard for calibration of full-field strain measurement using optical techniques, 'SPOTS' [15], currently implements only the in-plane strain calibration and detailed guidelines are given only for digital image correlation (DIC) and electronic speckle pattern interferometry (ESPI).

In this paper a calibration and correction procedure for out-of-plane shearography is proposed. Accurate rigid-body rotations of a flat plate, on the $\mu \mathrm{rad}$ level, are used to induce a constant displacement gradient. By comparing the known rotation of the plate and the displacement gradient measured by shearography, 2-dimensional maps, in this paper called the calibration maps, are computed. Calibration maps represent the systematic variability of the shearing distance. The shearing distance is induced by rotating (shearing) a mirror in the Michelson interferometer of the shearography camera. Since the lenses and the mirrors in the camera are not ideal, the induced shearing distance is not perfectly constant over the whole image. A calibration map, used to correct for this systematic error, is valid for a certain set of parameters of the shearography camera. A collection of calibration maps needs to be created to account for the possible settings of the shearography camera.

Depending whether the shearing distance is set in the $x$ or the $y$-direction, the displacement gradient in that direction is obtained. Practically, however, the shearing distance is difficult to control exactly and it commonly occurs that the measurements are done while both shearing distances are simultaneously non-zero. When both shearing distances are non-zero, even when one (the intended shearing distance) is much larger than the other (the unintended shearing distance) both displacement gradients are coupled in a single shearography measurement. In this paper the equations are derived which allow the two displacement gradients, in the $x$ - and the $y$-directions, to be decoupled from the shearography measurements, by utilizing two measurements with distinct shearing distances.

The correction procedure involves using the decoupling equations and the calibration maps to obtain the corrected shearography result. The correction procedure similarly takes into account the local value of the sensitivity vector. The concept of the shearing distance and the sensitivity vector are explained further in the text.

Two test cases for the calibration and correction procedure are developed - a rotation of a flat plate and a deformation of a centrally loaded flat plate. Shearography results of the second test case, with and without the correction procedure applied, are compared to a finite-element model (FEM) analysis.

The remainder of the introduction section gives a brief overview of the basic theory of shearography, common image processing in shearography and an overview of the error sources affecting shearography measurements. An overview and the interpretation of the literature regarding the error sources and the methods used to deal with them is also given. In the second section expressions are derived on how the displacement gradients are coupled in the resulting shearography images and the modified shearography equation is presented. The third section deals with the explanation of the optical distortions in the Michelson interferometer while the fourth section details the experiments used to obtain the calibration maps to correct for the optical distortions. The fifth section demonstrates how the correction procedure works, by decoupling the displacement gradients and applying the calibration maps to the two test cases.

\subsection{Principle of shearography}

Shearography always compares two different states of the surface to each other and is therefore called a double-exposure 
technique. Typically, this will involve comparing a deformed to an undeformed state. Deformation of the observed surface changes the optical path length of light from the laser source, reflected by the surface, to the detector of the shearography camera. In shearography, pairs of points, separated by the shearing distance, are used in such a way that the optical path length between the undeformed state and the deformed state quantifies the deformation gradient.

In this paper, for brevity, the basic shearography equations are given directly and not derived from first principles. For equation derivations see work of Hung and Liang [16] or, for more recent examples, a book by Steinchen and Yang [1] and the shearography overview by Francis et al [2].

The phase change, which is the quantity measured by shearography, is given by

$$
\Delta_{x}=\frac{2 \pi}{\lambda}\left(k_{x} \frac{\partial u}{\partial x}+k_{y} \frac{\partial v}{\partial x}+k_{z} \frac{\partial w}{\partial x}\right) \delta_{x}
$$

when the shearing distance $\delta_{x}$ is set in the $x$-direction. Analogously, when the shearing distance is set in the $y$-direction (in that case it is labelled as $\delta_{y}$ ), the phase change is

$$
\Delta_{y}=\frac{2 \pi}{\lambda}\left(k_{x} \frac{\partial u}{\partial y}+k_{y} \frac{\partial v}{\partial y}+k_{z} \frac{\partial w}{\partial y}\right) \delta_{y}
$$

The measured phase changes $\Delta_{x}$ or $\Delta_{y}$ depend on the wavelength of the illumination laser $\lambda$ and the components of the sensitivity vector $\mathbf{k}=\left\{k_{x}, k_{y}, k_{z}\right\}$. In this paper $x$ and $y$ label the in-plane axes and $z$ labels the out-of-plane axis. Displacements are $u, v$ and $w$ in the direction of the axes $x, y$ and $z$ respectively. Thus the $\partial\{u, v, w\} / \partial\{x, y\}$ are the six surface displacement gradients shearography can be made sensitive to. The sensitivity vector $\mathbf{k}$ is the sum of unit-vectors specimen-tocamera and specimen-to-source and $k_{x}, k_{y}$ and $k_{z}$ are its vector components in each of the coordinate system directions.

For an out-of-plane shearography setup, the light source and the camera are placed perpendicularly to the observed surface, approximately co-linearly. In that case the shearography setup is sensitive mostly to the out-of-plane displacement gradients, either $\partial w / \partial x$ or $\partial w / \partial y$, as the sensitivity components are $k_{x} \approx 0, k_{y} \approx 0$ and $k_{z} \approx 2$. The approximate out-of-plane shearography equations are thus

$$
\begin{aligned}
& \Delta_{x}=\frac{4 \pi}{\lambda} \frac{\partial w}{\partial x} \delta_{x} \\
& \Delta_{y}=\frac{4 \pi}{\lambda} \frac{\partial w}{\partial y} \delta_{y}
\end{aligned}
$$

The deformation gradient calculation is based on determining the optical path length. The method for actually determining the difference in the optical path length, between the undeformed and the deformed states, is based on measuring the phase difference of light. This principle can be illustrated by the schematic of the shearography camera in figure 2. A speckled wavefront is produced by the interference of coherent light, emitted by the laser and scattered by the diffuse object surface. The wavefront enters the shearography camera and first passes through an optical device called the Michelson interferometer. The Michelson interferometer superimposes two copies of a speckled wavefront, translated by the shearing distance $\delta_{x}$. This creates an interferometric speckle pattern on the camera detector, sensitive to the displacement gradient. The camera detector normally only registers the light intensity of the speckle pattern. By translating the phase-shifting mirror (for fractions of the wavelength $\lambda$ ) during the measurement and by using the associated computation algorithms, the phase difference $\Phi$ can be additionally computed. More details on this, the phase-shifting technique, can be found in $[1,2]$. Typically shearography captures two phase-differences- $\Phi_{1}$ for the undeformed state and $\Phi_{2}$ for the deformed state. A subtraction of the phase differences results in the phase change

$$
\Delta_{x}=\Phi_{2}-\Phi_{1} \quad \text { or } \quad \Delta_{y}=\Phi_{2}-\Phi_{1}
$$

depending on whether the shearing distance between points is set in the $x$-direction or in the $y$-direction. From the phase change $\Delta_{x}$ the surface displacement gradients can be computed using equations $(1 a)$ or $(1 b)$.

\subsection{Image processing in shearography}

The equations (1) in the previous subsection are derived for a single point in the image plane of the camera detector. Shearography, however, is a full-field technique producing a 2-dimensional field of values $\Delta(x, y)$, in the so-called $\Delta$ image. The undeformed and the deformed states are captured as phase difference fields, the $\Phi$-images. The steps for the shearography image acquisition are thus:

(a) the initial phase difference field is acquired as $\Phi_{1}(x, y)$

(b) the surface is deformed or displaced

(c) the resulting phase difference field is acquired as $\Phi_{2}(x, y)$

(d) the displacement gradient is calculated as $\Delta(x, y)=$ $\Phi_{2}(x, y)-\Phi_{1}(x, y)$.

1.2.1. Unwrapping. The $\Phi$-images are in the interval $[0,2 \pi)$ as a phase difference of a light wave can only be defined in this interval. $\Delta$-images, as a subtraction of two $\Phi$-images, are defined in the interval $[-\pi, \pi)$. As the value of a deformation gradient is rising and approaching the limit of the interval, it cannot surpass it, but it gets 'wrapped' back to the bottom of the interval. In other words, the measured phase difference values are always shifted into the $[-\pi, \pi)$ interval by an integer multiple of $2 \pi$. The wrapping function $W$ can be written as $W(\Delta)=\Delta+2 n \pi$ where $W(\Delta) \in[-\pi, \pi)$ and $n$ is an integer number. This results in the fringes in the shearography images, where the $2 \pi$ jumps are present. Unwrapping algorithms [17] locate the jumps and correct the integer number $n$ of the $2 \pi$ shifts to create a smooth surface. For qualitative analysis unwrapping is often not necessary, but for quantitative analysis it is required.

1.2.2. Denoising. Similar to other interferometric techniques, shearography is affected by a high level of noise. Noise is detrimental for qualitative image analysis just as much as for quantitative analysis. Unwrapping algorithms often only tolerate a very small amount of noise, so before they can be applied, 


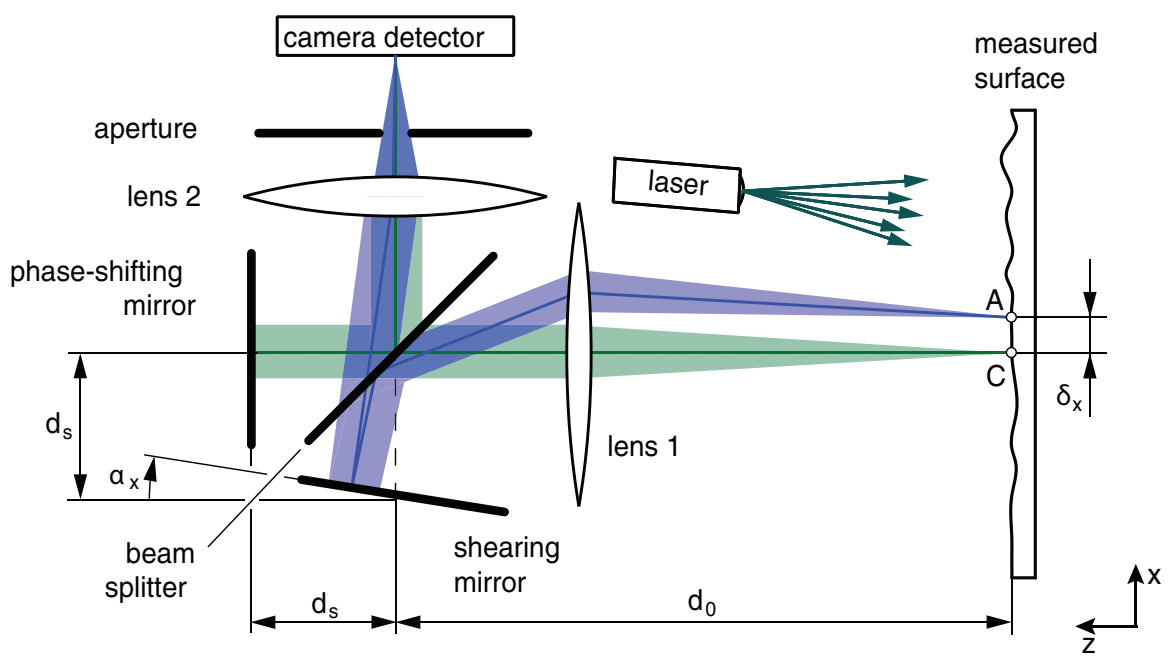

Figure 2. The Michelson interferometer is the most common shearing device for shearography. Two points from the surface are superimposed onto the same point on the camera detector due to a rotation of a mirror by a shearing angle $\alpha_{x}$.

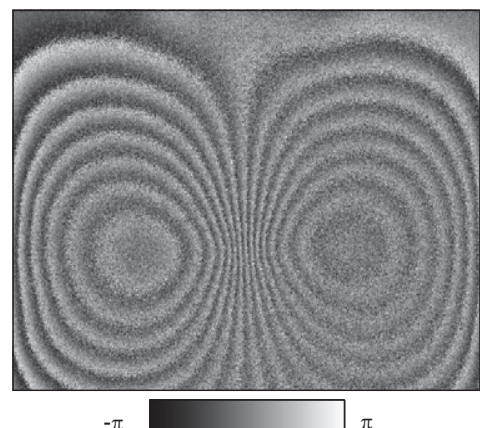

(a)

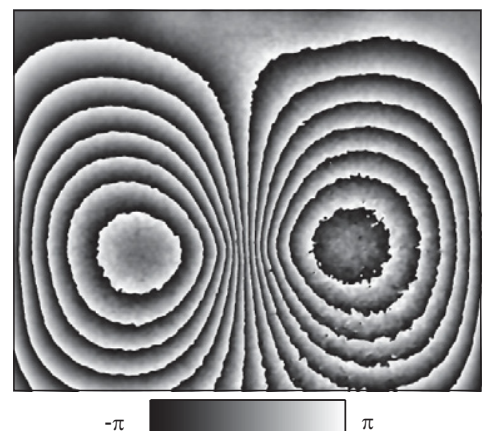

(b)

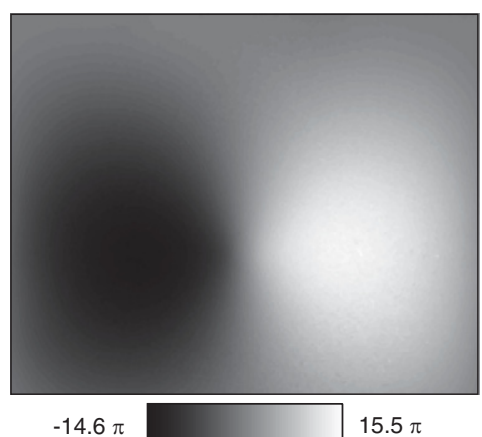

(c)

Figure 3. To be used qualitatively, a $\Delta$-image $(a)$ needs to have noise removed by filtering $(b)$ and unwrapping performed $(c)$ to remove the fringes.

denoising is necessary. Noise can be removed in shearography using filtering methods. Some common filtering methods are the Gaussian filter, the averaging filter and the median filter, described in detail in [1]. An effective technique for filtering interferometric phase fringe patterns is proposed by Aebischer and Waldner [18]. In figure 3 the image processing steps from a raw $\Delta$-image, the filtering and the unwrapping, are shown. In figures $3(a)$ and $(b)$ the fringes are visible, before the unwrapping is performed.

\subsection{Errors in shearography}

The errors in shearography discussed in literature include the (i) errors due to the linearisation of the shearography equations, (ii) errors due to the environmental disturbances, (iii) errors occurring in the phase reconstruction algorithms, (iv) errors when assuming constant sensitivity vectors, (v) measurement error of the shearing distance and the errors from the assumption of the constant shearing distance, (vi) errors related to the stability of the components of the measurement setup, (viii) errors due to the rigid-body translation and rotation during measurement. These error sources are explained and reviewed one-by-one in the following paragraphs.

(i) When deriving the basic equations (1) the higher order terms of the displacements are neglected. Thus in shearography the gradients are computed using the linearised displacements. Equation linearisation was discussed as a systematic error source in shearography calculations by Fulton et al [19] and by Steinchen et al [20]. The conclusion drawn in these papers was that the errors are negligible, the relative error being less than $1 \%$, except in extreme cases. These extreme cases are

- when the shearing distances are large, $\left\{\delta_{x}, \delta_{y}\right\} \gg 1 \mathrm{~cm}$, - when the camera detector or the laser source is close to the specimen, $\left\{z_{D}, z_{S}\right\}<10 \mathrm{~cm}$,

- when the ratio of the first order gradients of the deformation to the second order gradients is much larger than the shearing distance, $\max \left|\frac{\partial\{u, v, w\}}{\partial\{x, y\}}\right| / \max \left|\frac{\partial^{2}\{u, v, w\}}{\partial\{x, y\}^{2}}\right|>50\left\{\delta_{x}, \delta_{y}\right\}$.

(ii) Environmental disturbances (the environmental vibrations and the air-currents) play a major role in all measurement 
techniques involving interferometry. Shearography is generally considered to be less susceptible to environmental vibrations than the other interferometric techniques, as the gradient measurement is not affected by the rigidbody translations. However, our previous work [13, 14, 21] has shown that vibration isolation can greatly benefit noise reduction in the measurements. The refraction between zones of air with different temperatures can cause transient optical distortion. Thus the shearography measurements are affected by the air-currents, especially the warm air rising between the camera, the laser and the observed surface. The environmental disturbances can be considered random and their influence is increased with the duration of the experiments [21]. The high-frequency noise is related to the laser speckle and the speckle decorrelation and the influence of it can be reduced by filtering. The low-frequency noise due to the air-currents is not affected by filtering, but averaging the results of several identical measurements can reduce the influence of the random environmental influences.

(iii) The phase reconstruction is the step in the shearography measurement where the $\Phi$-image is calculated from intensity images captured by the camera detector. The phase reconstruction is made from three or more intensity images captured when the phase-shifting mirror (shown in figure 2) is displaced by a fraction of the wavelength. More detailed explanation of the phase-stepping to obtain the shearography images can be found in the book by Steinchen and Yang [1] and the review of shearography by Francis et al [2]. The lack of accuracy in the displacement of the phase-shifting mirror can cause errors in the measurements. However, the phase-reconstruction algorithms, such as the one developed by Carré [22, 23], Hariharan et al [24] and Joenathan [25], do not need calibrated phaseshifts to accurately reconstruct the phase. An overview of the available phase-reconstruction algorithms and the associated errors is given by Hack and Burke [26].

(iv) The local value of the sensitivity vector $\mathbf{k}=\left\{k_{x}, k_{y}, k_{z}\right\}$ in general varies over the observed surface. When using the simplified shearography equations (2), the calculation of the local value of the sensitivity vector across the field-of-view is altogether neglected. The errors due to neglecting the variation of the sensitivity vector were discussed in detail by Farrant and Petzing [27]. It is reported that the measurement error of the displacement gradient, if the sensitivity vector is neglected, can be up to $11 \%$ for the out-of-plane shearography configurations. The local value of the sensitivity vector can be calculated from the relative positions of the camera and the laser as compared to the position and the size of the observed surface. Still, in most research published on quantitative shearography, the calculation of the local sensitivity vector is not mentioned. Works that mention calculating the local sensitivity vector include [28-31].

(v) As shown by equations (1), the measured gradients of deformation $\partial w / \partial x$ and $\partial w / \partial y$ are directly affected by the shearing distances $\delta_{x}$ and $\delta_{y}$. Thus an accurate measurement of the shearing distance is crucial to obtain accurate results from shearography. $\mathrm{Ng}$ [32] proposed calculation of the shearing distance from the cross correlation between the two separately acquired images. The two images were acquired by first obscuring one and then the other mirror in the Michelson interferometer, thus obtaining separate images for the sheared and the non-sheared images. A similar process was proposed by Andersson et al [33], but digital speckle photography was used to calculate the resulting shearing distance instead of the cross-correlation. Both methods require modifications to the Michelson interferometer and are limited to the accuracy of 1 pixel. An uncertainty of 1 pixel, when the typical shearing distance is 20 to 40 pixels, is analogous to a measurement uncertainty of $2.5 \%$ to $5 \%$. Lee et al [34] used a more accurate approach. A pattern of stripes, with sinusoidally varying brightness, is imaged over each of the two mirrors in the Michelson interferometer separately. Since the shearing distance is calculated from the change in brightness, it is not limited to the single pixel resolution.

However, the shearing distance does not need to be constant over the whole field-of-view. The shearing distances $\delta_{x}$ and $\delta_{y}$ for a point are proportional to the shearing angles $\alpha_{x}$ and $\alpha_{y}$, respectively, and to the distance from the camera to that point. The shearing distances $\delta_{x}$ and $\delta_{y}$ are thus approximately constant for a flat plate perpendicular to the camera as all the points on a flat plate are approximately equally distant from the camera. For a non-flat surface the shearing distance will necessarily vary, which was researched by Goto and Groves [31]. Even for a flat surface the shearing distance obtained from a Michelson interferometer was shown to vary by Aebischer and Rechsteiner [35] as the shearing induced by the Michelson interferometer is not a pure in-plane translation of the image but also incorporates rotation. The error predicted by Aebischer and Rechsteiner is very small, in the range of $<0.1 \%$ in a typical shearography configuration. Variation of the shearing distance due to the optical distortions in the Michelson interferometer is also possible, as the sheared and the non-sheared copies of an image are reflected over different mirrors. Goto and Groves [31] experimentally measured the shearing distance by a Michelson interferometer which was adapted so it could sequentially block either one of its two mirrors. Thus the sheared and the non-sheared images of the object covered in a random pattern could be acquired separately. A modified digital image correlation (DIC) algorithm was used to extract the shearing distance field from the random speckle pattern. The result of Goto and Groves [31] showed very large variation of the shearing distance (exact range for the shearing distance variation was not given explicitly). In a similar manner, using a modified DIC algorithm and sequential blocking of mirrors, Francis et al [36] experimentally measured a shearing variation for a shearography setup which included the Mach-Zehnder instead of the Michelson interferometer. The reported shearing variations across the image were as large as $10 \%$ ( $\pm 5 \%$ from the average value). 
(vi) The shearing distances $\delta_{x}$ and $\delta_{y}$ are induced by rotating the shearing mirror by angles $\alpha_{x}$ and $\alpha_{y}$. This rotation can be actuated by a screw, or by a piezo-element (most often PZT material is used). In commercial shearography systems the piezo-element actuated shearing mirror is practical as it allows the shearing to be controlled remotely. The PZT material can exhibit creep behaviour, which can affect the measurement. Research by our group [21] showed that the PZT creep can create a considerable error in the measurements. This error is more pronounced with increasing duration of the measurement, so it is important mostly for long time-span measurements.

(vii) The effects of rigid-body translations and rotations are partially suppressed in shearography due to the fact that both interfering beams share the same path. The rigidbody effects in shearography were studied by Hack [37]. In this paper, however, the rigid-body rotations are exploited in the process of calibration.

Due to the error sources described here, accurate measurements are difficult to achieve with shearography. Recently, some researchers directly compared the results from quantitative measurements using shearography to the results from finite element analyses and to those from other measurement techniques. Goto and Groves [31] investigated an aluminium cylinder loaded by an internal pressure and the computed strains were compared to the finite element analysis. The strains showed discrepancy between the experiment and the finite element analysis in the interval of $[5 \%, 50 \%]$ for longitudinal strains and around 5\% for the tangential strains. Rosso et al [38] investigated an out-of-plane shearography experiment. A plate centrally loaded by a weight was compared to the finite element analysis, resulting in a displacement gradient discrepancy of up to $12 \%$. Another paper by Groves et al [10] compares measurements of a hydrostatically loaded PVC pipe with shearography, fibre Bragg sensors and resistive foil strain gauges. The discrepancy of the measured strain, between the shearography and the resistive foil strain gauges was, respectively for axial and hoop strains, up to $10 \%$ and $6 \%$. The discrepancy between shearography and the prediction from analytic relations for a hydrostatically loaded cylinder was up to $1 \%$ and $12 \%$. The reported discrepancies between the different techniques were not further analyzed.

In this paper, primary emphasis is placed on the calibration of the local shearing distance in the Michelson interferometer. The local variation of the shearing distance is experimentally determined in the calibration procedure and the so-called calibration maps are generated. These calibration maps are used in the correction procedure along with the local variation of the sensitivity vector. A third thing encompassed in the correction procedure is the gradient coupling, which is described in the following section.

\section{Effect of the gradient coupling}

The shearography equations are commonly derived for shearing in one of the two perpendicular directions, as shown in equations (1). Each of these equations assumes the shearing

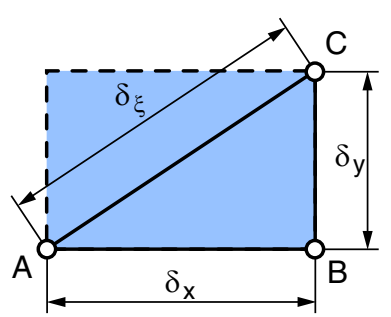

Figure 4. When both shearing distances $\delta_{x}$ and $\delta_{y}$ are present, the actual deformation gradient is obtained in the $\xi$-direction, as shearing distance $\delta_{\xi}$.

distance is only in one direction, with the shearing distance in the perpendicular direction zero. But in real shearography experiments, the unwanted shearing distance in the perpendicular direction is often small, but not equal to zero. The common procedure to set the shearing distance involves first setting the zero shearing distance in $x$ - and $y$-directions and then the desired shearing distance, either $\delta_{x}$ or $\delta_{y}$. During this procedure there is only visual feedback from the shearography image so the zero shearing is difficult to set exactly. Additionally, the axes of the shearing mirror in the Michelson interferometer can be slightly misaligned to the camera detector axes, thus setting the shearing distance in one direction can affect the other (perpendicular) one. In order to negate this effect, both shearing distances $\delta_{x}$ and $\delta_{y}$ are measured before acquiring shearography images and equations are derived to decouple their influence.

When shearing is present in both the $x$ - and the $y$-directions, the shearography measures the deformation gradients $\frac{\partial\{u, v, w\}}{\partial \xi}$, in the direction $\xi$. The direction $\xi$ is defined by the the unit vector $\hat{\xi}=\left(\delta_{x} \hat{x}+\delta_{y} \hat{y}\right) / \delta_{\xi}$ where $\hat{x}$ and $\hat{y}$ are unit vectors in the $x$ and $y$ directions and $\delta_{\xi}=\sqrt{\delta_{x}^{2}+\delta_{y}^{2}}$. The shearography equation should then be written as

$$
\Delta=\frac{2 \pi}{\lambda}\left(k_{x} \frac{\partial u}{\partial \xi}+k_{y} \frac{\partial v}{\partial \xi}+k_{z} \frac{\partial w}{\partial \xi}\right) \delta_{\xi}
$$

In the general case, the gradients of deformation $\partial\{u, v$, $w\} / \partial\{x, y\}$ cannot be calculated from the gradients $\partial\{u, v$, $w\} / \partial \xi$, unless first the deformation surface is reconstructed from its gradients. In shearography, however, the gradients of deformation are approximated. The approximation for the gradients of deformation is calculated from the relative displacements of points on the surface. This makes it possible to reconstruct what the deformation gradient would be if the shearing distance was only in the $x$ - or $y$-direction. The gradient of deformation is calculated from within a region of size $\delta_{x} \times \delta_{y}$. Consider the out-of-plane deformations $w_{A}, w_{B}$ and $w_{C}$ of the points $\mathrm{A}, \mathrm{B}$ and $\mathrm{C}$ in figure 4 . The actual measured gradient of deformation is approximated as

$$
\frac{\partial w}{\partial \xi}=\frac{w_{C}-w_{A}}{\delta_{\xi}}
$$

The gradients $\partial w / \partial\{x, y\}$, which are sought, can be defined from figure 4 as 


$$
\begin{aligned}
& \frac{\partial w}{\partial x}=\frac{w_{B}-w_{A}}{\delta_{x}} \\
& \frac{\partial w}{\partial y}=\frac{w_{C}-w_{B}}{\delta_{y}}
\end{aligned}
$$

Analogous to equations (4) and (5) for the out-of-plane displacement $w$, the in-plane equations for $u$ and $v$ can be established. Combining these equations produces

$$
\frac{\partial\{u, v, w\}}{\partial \xi} \delta_{\xi}=\frac{\partial\{u, v, w\}}{\partial x} \delta_{x}+\frac{\partial\{u, v, w\}}{\partial y} \delta_{y}
$$

Equation (3) can be expanded by the use of the equation (6) leading to an equation for the $\Delta$-image with contributions from both deformation gradients in the $x$ - and the $y$-directions

$$
\begin{aligned}
\Delta= & \frac{2 \pi}{\lambda}\left(k_{x} \frac{\partial u}{\partial x}+k_{y} \frac{\partial v}{\partial x}+k_{z} \frac{\partial w}{\partial x}\right) \delta_{x} \\
& +\frac{2 \pi}{\lambda}\left(k_{x} \frac{\partial u}{\partial y}+k_{y} \frac{\partial v}{\partial y}+k_{z} \frac{\partial w}{\partial y}\right) \delta_{y}
\end{aligned}
$$

For out-of-plane shearography, when the in-plane sensitivity components $k_{x}$ and $k_{y}$ are close to zero and the out-of-plane displacements are dominant over the in-plane displacements $\partial w / \partial\{x, y\} \gg \partial\{u, v\} / \partial\{x, y\}$ the equation can be written as

$$
\Delta=\frac{2 \pi}{\lambda} k_{z}\left(\frac{\partial w}{\partial x} \delta_{x}+\frac{\partial w}{\partial y} \delta_{y}\right)
$$

Disregarding the influence of the perpendicular shearing distance causes the measurement error to be proportional to the ratio of the shearing distances, $\delta_{y} / \delta_{x}$ when $\delta_{x}$ is the dominant one and $\delta_{x} / \delta_{y}$ when $\delta_{y}$ is the dominant one. The ratio of the two displacement gradients also directly influences the error, but that ratio is not fixed. As an example, provided the deformation gradients are of similar magnitude, for a shearing distance $\delta_{x}=5 \mathrm{~mm}$, a parasitic shearing distance $\delta_{y}=0.2 \mathrm{~mm}$ causes an error of $4 \%$.

Decoupling the two gradients from a single measurement is not possible. However, if two equations with two distinct pairs of shearing distances are obtained, such that

$$
\begin{aligned}
& \Delta_{1}=\frac{2 \pi}{\lambda}\left(k_{z} \frac{\partial w}{\partial x} \delta_{x 1}+k_{z} \frac{\partial w}{\partial y} \delta_{y 1}\right) \\
& \Delta_{2}=\frac{2 \pi}{\lambda}\left(k_{z} \frac{\partial w}{\partial x} \delta_{x 2}+k_{z} \frac{\partial w}{\partial y} \delta_{y 2}\right)
\end{aligned}
$$

the displacement gradients can be decoupled as

$$
\begin{aligned}
& \frac{\partial w}{\partial x}=\frac{\lambda}{2 \pi k_{z}} \frac{\Delta_{1} \delta_{y 2}-\Delta_{2} \delta_{y 1}}{\delta_{x 1} \delta_{y 2}-\delta_{x 2} \delta_{y 1}} \\
& \frac{\partial w}{\partial y}=\frac{\lambda}{2 \pi k_{z}} \frac{\Delta_{2} \delta_{x 1}-\Delta_{1} \delta_{x 2}}{\delta_{x 1} \delta_{y 2}-\delta_{x 2} \delta_{y 1}}
\end{aligned}
$$

The shearing distances for measurement $1\left(\delta_{x 1}\right.$ and $\left.\delta_{y 1}\right)$ and for measurement $2\left(\delta_{x 2}\right.$ and $\left.\delta_{y 2}\right)$ need to be separately measured to use equations (9). Notice that in the case $\delta_{y 1}=0, \delta_{x 2}=0$, $\delta_{x 1}=$ const, $\delta_{y 2}=$ const and $k_{z}=2$ equations (9) simplify into equation (2).

\section{Optical distortions in the Michelson interferometer}

As shown in figure 2, the Michelson interferometer, consisting of two mirrors and a beam splitter, is surrounded by two convergent lenses and an aperture. Like any optical system, it is not perfect. Surface imperfections of the components, like the deviation from flatness, can cause very small optical distortion. Due to the fact that shearography measures such smallscale deformations, these distortions can contribute to an appreciable systematic error.

The value of the shearing distances $\delta_{x}$ and $\delta_{y}$ in the Michelson interferometer are controlled by the associated shearing angles $\alpha_{x}$ and $\alpha_{y}$. A light beam from the sheared point $\mathrm{A}$ in figure 2 passes through the lens 1 , reflects from the bottom side of the beam splitter, reflects from the angled shearing mirror, passes through the beam splitter and is focused by the lens 2 onto the camera detector. A light beam from the non-sheared point $\mathrm{C}$ travels over a different path, passing through the lens 1, passing through the beam splitter, reflecting from the phase-shifting mirror, reflecting from the top side of the beam splitter and is focused by the lens 2 onto the camera detector. Any passage through a lens or a reflection from a mirror can induce a slight deviation of the beam's angle, as compared to a perfect lens or a mirror. These deviation angles will sum over the whole path of the beams through the Michelson interferometer. The deviation depends on where a beam is entering the Michelson interferometer and at what angle.

Light from a surface point does not travel along a single path to the camera detector. Instead, a bundle of light beams is guided through the Michelson interferometer onto the camera detector. The light over the whole light bundle is focused onto the same point of the camera detector so the value measured by the camera detector is actually averaged over the cross-section area of a bundle. The deviations will be less pronounced if the light bundle is wider as the distortions are also averaged over the cross-section area of the bundle. The light beam bundle cross-section area is regulated by the opening of the aperture $S_{A}$. The wider the opening of the aperture is, the wider the bundles are. However, the aperture regulates a multitude of other parameters in the shearography setup. The aperture regulates the light intensity that passes onto the camera detector and by that the brightness of the image. Below the minimum practical aperture opening, the brightness is not sufficient to obtain a $\Delta$-image even when using the highest illumination power of the laser. The laser speckle size is also related to the opening of the aperture. Larger aperture opening will cause smaller speckles, so the maximal aperture opening is limited by the smallest allowable speckle size of one pixel. The aperture must thus be set within a certain range to be practically usable in shearography. The different settings of the aperture are not directly measurable in the shearography setup. The aperture 
change is calculated from the change in the power of the laser source needed to keep the brightness in the image constant.

One point on the camera detector superimposes light from two points from the measured surface. When there are no optical distortions the pairs of points on the surface would be separated by a shearing distance proportional to the shearing angle. When there are optical distortions the shearing distance between the pairs of points is varying and is no longer proportional to the shearing angle. Despite a disproportionate relation between the varying shearing distance and a shearing angle, one point on the camera detector is still related to exactly two points on the measured surface. A varying shearing angle is thus used to model the phenomenon causing optical distortions in the Michelson interferometer. The varying shearing angle is composed of the physical angles of the shearing mirror $\alpha_{x}$ and $\alpha_{y}$ and the spatially varying components $\alpha_{C x}(x, y)$ and $\alpha_{C y}(x, y)$. In this paper, the spatially varying components $\alpha_{C x}(x, y)$ and $\alpha_{C y}(x, y)$ are called the calibration maps. Using the calibration maps, the distortion of the whole optical system, encompassing the Michelson interferometer, the lenses and the aperture, is modelled. As the calibration maps show the cumulative effect of the optical distortions, a separate effect of each optical component cannot be isolated. The calibration maps $\alpha_{C x}(x, y)$ and $\alpha_{C y}(x, y)$ depend on the shearing angles $\alpha_{x}$ and $\alpha_{y}$ and the aperture opening $S_{A}$.

Aebischer and Rechsteiner [35] defined the two perpendicular shearing distances $\delta_{x}$ and $\delta_{y}$ as

$$
\begin{aligned}
& \delta_{x}=\left(d_{0}+d_{s}\right) \sin 2 \alpha_{x}+2 x \sin ^{2} \alpha_{x} \\
& \delta_{y}=\left(d_{0}+d_{s}\right) \sin 2 \alpha_{y}+2 y \sin ^{2} \alpha_{y}
\end{aligned}
$$

where, as shown in figure $2, d_{0}$ is the distance from the observed surface to the center of the Michelson interferometer, $d_{s}$ the distance from the center of the Michelson interferometer to the center of the shearing mirror. $x$ and $y$ are the coordinates of a point in the image. Equation (10) does not take optical distortion into account, but still, it predicts a variation of the shearing distance over the image.

The shearing angles are allowed to vary over the field-ofview to account for the optical distortions and are represented as $\alpha_{x}(x, y)=\overline{\alpha_{x}}+\widetilde{\alpha_{x}}(x, y)$ and $\alpha_{y}(x, y)=\overline{\alpha_{y}}+\widetilde{\alpha_{y}}(x, y)$, the sums of the average shearing angles $\left(\overline{\alpha_{x}}\right.$ and $\left.\overline{\alpha_{y}}\right)$ and the variation around the average values $\left(\widetilde{\alpha_{x}}\right.$ and $\left.\widetilde{\alpha_{y}}\right)$. For the shearing in the $x$-direction equation (10) becomes

$$
\begin{aligned}
\delta_{x}(x, y)= & \left(d_{0}+d_{s}\right) \sin 2\left[\overline{\alpha_{x}}+\widetilde{\alpha_{x}}(x, y)\right] \\
& +2 x \sin ^{2}\left[\overline{\alpha_{x}}+\widetilde{\alpha_{x}}(x, y)\right]
\end{aligned}
$$

The average shearing angle is a small angle, on the order of magnitude of mrad, but the shearing distance variation is even several orders of magnitude smaller. Thus the equation is simplified using $\cos 2 \widetilde{\alpha_{x}} \approx 1$ and $\sin 2 \widetilde{\alpha_{x}} \approx 2 \widetilde{\alpha_{x}}$ :

$$
\begin{aligned}
\delta_{x}(x, y)= & \left(d_{0}+d_{s}\right) \sin 2 \overline{\alpha_{x}}+2\left(d_{0}+d_{s}\right) \widetilde{\alpha_{x}}(x, y) \cos 2 \overline{\alpha_{x}} \\
& +2 x \sin ^{2}\left[\overline{\alpha_{x}}+\widetilde{\alpha_{x}}(x, y)\right]
\end{aligned}
$$

The average shearing distance is introduced to account for the spatially constant part of equation (11) as

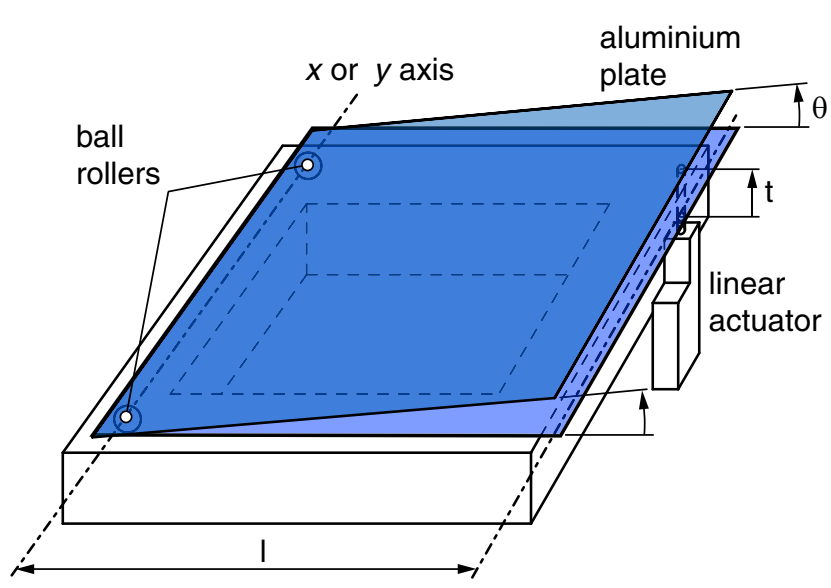

Figure 5. The mechanism for the rigid-body rotation of a flat plate Displacement $t$ of a linear actuator at a distance $l$ from the rotation axis induces rotation angle $\theta$.

$$
\overline{\delta_{x}}=\left(d_{0}+d_{s}\right) \sin 2 \overline{\alpha_{x}}
$$

The subsequent terms in equation (11) are related to the spatially varying part of the shearing distance and they will be experimentally measured as the calibration map $\alpha_{C x}(x, y)$

$$
\begin{aligned}
& 2\left(d_{0}+d_{s}\right) \alpha_{C x}(x, y)=2\left(d_{0}+d_{s}\right) \widetilde{\alpha_{x}}(x, y) \cos 2 \overline{\alpha_{x}} \\
& \quad+2 x \sin ^{2}\left[\overline{\alpha_{x}}+\widetilde{\alpha_{x}}(x, y)\right]
\end{aligned}
$$

The calibration map $\alpha_{C x}$ varies over the $x$ - and $y$-coordinates and is dependent on the shearing angles $\alpha_{x}$ and $\alpha_{y}$ and the aperture opening $S_{A}$. A similar procedure can be performed for the sharing distance $\delta_{y}$, thus obtaining a pair of equations

$$
\begin{aligned}
& \delta_{x}(x, y)=\overline{\delta_{x}}+2\left(d_{0}+d_{s}\right) \alpha_{C x}(x, y) \\
& \delta_{y}(x, y)=\overline{\delta_{y}}+2\left(d_{0}+d_{s}\right) \alpha_{C y}(x, y)
\end{aligned}
$$

In the next section the varying shearing distance is experimentally measured and the relation to the optical parameters in the Michelson interferometer is shown.

\section{Calibration of the optical distortions using the rigid-body rotations}

\subsection{Calibration equations}

The calibration is performed using a flat plate that can be rotated around the $x$-axis by a small angle $\theta_{x}$, or around the $y$-axis by $\theta_{y}$. The mechanism is shown in figure 5 . The rotation will produce a uniform field $\partial w / \partial y$ or $\partial w / \partial x$, respectively, when imaged with an out-of-plane shearography setup. If the rotation angle $\theta_{x}$ or $\theta_{y}$ is known with high precision, the variation in the $\Delta$-image can be used for calibration.

First, consider the case of rotation around the $y$-axis. Since the rotation angle $\theta_{y}$ is small, the in-plane gradient components are negligible as $\partial\{u, v\} / \partial x \ll \partial w / \partial x$. In the experiment it is also imposed that $\delta_{x} \gg \delta_{y}$ and by accurately setting the rotation around the $y$-axis only, $\partial\{u, v, w\} / \partial y=0$, equation (7) simplifies into 


$$
\Delta_{x}=k_{z} \frac{2 \pi}{\lambda} \frac{\partial w}{\partial x} \delta_{x}
$$

The phase change $\Delta_{x}$, the deformation gradient $\partial w / \partial x$, the out-of-plane sensitivity vector $k_{z}$ and the shearing distance $\delta_{x}$ are fields of values and they depend on the $x$ - and $y$-coordinates, so the previous equation can be written as

$$
\Delta_{x}(x, y)=k_{z}(x, y) \frac{2 \pi}{\lambda} \frac{\partial w(x, y)}{\partial x} \delta_{x}(x, y)
$$

Introducing equation (13) yields

$$
\Delta_{x}(x, y)=k_{z}(x, y) \frac{2 \pi}{\lambda} \frac{\partial w(x, y)}{\partial x}\left[\overline{\delta_{x}}+2\left(d_{0}+d_{s}\right) \alpha_{C x}(x, y)\right]
$$

When the measured surface is rotated as a rigid body by an angle $\theta_{y}$, its gradient of displacement will be constant over the whole field of view $\partial w(x, y) / \partial x=\theta_{y}$. The calibration map $\alpha_{C x}$ can be calculated as

$\alpha_{C x}(x, y)=\frac{1}{2\left(d_{0}+d_{s}\right)} \frac{\Delta_{x}(x, y)-k_{z}(x, y) \theta_{y} \overline{\delta_{x}} 2 \pi / \lambda}{k_{z}(x, y) \theta_{y} 2 \pi / \lambda}$

Similarly, for the rigid-body rotation around the $x$-axis by an angle $\theta_{x}$, the conditions $\partial\{u, v\} / \partial y \ll \partial w / \partial y, \delta_{y} \gg \delta_{x}$ and $\partial\{u, v, w\} / \partial x=0$ apply, simplifying equation (7) into

$$
\Delta_{y}=k_{z} \frac{2 \pi}{\lambda} \frac{\partial w}{\partial y} \delta_{y}
$$

From $\partial w(x, y) / \partial y=\theta_{x}$ and equation (13) the calibration map $\alpha_{C y}$ can be calculated as

$\alpha_{C y}(x, y)=\frac{1}{2\left(d_{0}+d_{s}\right)} \frac{\Delta_{y}(x, y)-k_{z}(x, y) \theta_{x} \overline{\delta_{y}} 2 \pi / \lambda}{k_{z}(x, y) \theta_{x} 2 \pi / \lambda}$

There is uncertainty associated with both the prescribed angles, $\theta_{x}$ and $\theta_{y}$, and the measured average shearing distances, $\overline{\delta_{x}}$ and $\overline{\delta_{y}}$. The averaged phase changes over the image are introduced as

$$
\begin{aligned}
& \overline{\Delta_{x}}=\operatorname{mean}\left[\Delta_{x}(x, y)\right]=\overline{k_{z}} \frac{2 \pi}{\lambda} \overline{\delta_{x}} \operatorname{mean}\left(\frac{\partial w}{\partial x}\right) \\
& \overline{\Delta_{y}}=\operatorname{mean}\left[\Delta_{y}(x, y)\right]=\overline{k_{z}} \frac{2 \pi}{\lambda} \overline{\delta_{y}} \operatorname{mean}\left(\frac{\partial w}{\partial y}\right)
\end{aligned}
$$

where $\overline{k_{z}}$ is the averaged out-of-plane sensitivity. The rotation angles $\theta_{y}$ and $\theta_{x}$ are constant so equations

$$
\begin{aligned}
& \theta_{y}=\frac{\lambda}{2 \pi \overline{k_{z}} \overline{\delta_{x}}} \overline{\Delta_{x}} \\
& \theta_{x}=\frac{\lambda}{2 \pi \overline{k_{z}} \overline{\delta_{y}}} \overline{\Delta_{y}}
\end{aligned}
$$

should hold. In that case equations (14) and (15) can be transformed into

$$
\begin{aligned}
& \alpha_{C x}(x, y)=\frac{\overline{\delta_{x}}}{2\left(d_{0}+d_{s}\right)} \frac{\Delta_{x}(x, y)\left[\overline{k_{z}} / k_{z}(x, y)\right]-\overline{\Delta_{x}}}{\overline{\Delta_{x}}} \\
& \alpha_{C y}(x, y)=\frac{\overline{\delta_{y}}}{2\left(d_{0}+d_{s}\right)} \frac{\Delta_{y}(x, y)\left[\overline{k_{z}} / k_{z}(x, y)\right]-\overline{\Delta_{y}}}{\overline{\Delta_{y}}}
\end{aligned}
$$

which are independent of the uncertainty of $\theta_{x}, \theta_{y}, \overline{\delta_{x}}$ and $\overline{\delta_{y}}$.

Using equations (17), the mean measured value is used to calibrate the relative shearing variation over the field-ofview of the camera. This is valid only if the global calibration, shown in equations (16), is first experimentally established. In the following paragraphs it will be shown that the uncertainty of setting the angles $\theta_{x}$ and $\theta_{y}$, even using a high-precision actuator, is higher than that of measuring the averaged phase changes $\Delta_{x}$ and $\Delta_{y}$. Thus using the averaged phase changes $\Delta_{x}$ and $\Delta_{y}$ is the preferred way of calibrating the relative shearing distance variation as it will result in lower uncertainty.

\subsection{Measurement setup}

The setup is shown in figure 6 . The rigid-body rotation of a $400 \times 400 \times 5 \mathrm{~mm}^{3}$ aluminium plate is measured. The plate is lying on top of a rigid vertical frame, built of extruded T-slotted aluminium profiles with a $40 \times 40 \mathrm{~mm}^{2}$ cross-section. The shearography camera is fixed on the bottom of the frame, looking upward. The plate is flat and machined to have an even surface.

The shearography camera used is a commercial shearography system SE3-D145 from manufacturer isi-sys, Germany. The sensor of the camera is a Sony ICX285 2/3" CCD with $\mathrm{XGA}+$ resolution $(1392 \times 1040$ pixels $), 8$-bit digitalization level and maximum acquisition rate of $15 \mathrm{~Hz}$. The laser source is an isi-sys LD-320 $4 \times 80 \mathrm{~mW}$ diode array producing light at $\lambda=658 \mathrm{~nm}$ (nominal centre wavelength). Internal lenses have a focal length of $50 \mathrm{~mm}$. The image acquisition software is the isi-sys Studio 2011.

The shearography system, the frame, the plate and its rotation mechanism are placed on an active vibration-isolating table. The table is pneumatically actuated and limits the environmental vibration during measurements, which is critical for obtaining good signal-to-noise ratio in the shearography measurements. Shearography requires the imaged surface to be diffusely reflective, so the face of the plate observed with shearography is coated with white paint. The experiments are performed in a temperature-stable environment and the movement of the persons around the equipment is limited during the experiments.

As the operation of the laser and the piezo-electric elements are affected by the temperature, the setup is left to stabilize for $30 \mathrm{~min}$ or more after any major change of settings. In the shearography camera, the change in the shearing distance can be performed either mechanically or by piezo-electric elements. It was shown previously [21] that using the piezo-elements to control the shearing distance can cause measurement errors due to the creep response of the piezo-electric material. Thus, for the purpose of the experiments in this paper, the shearing distance was induced 


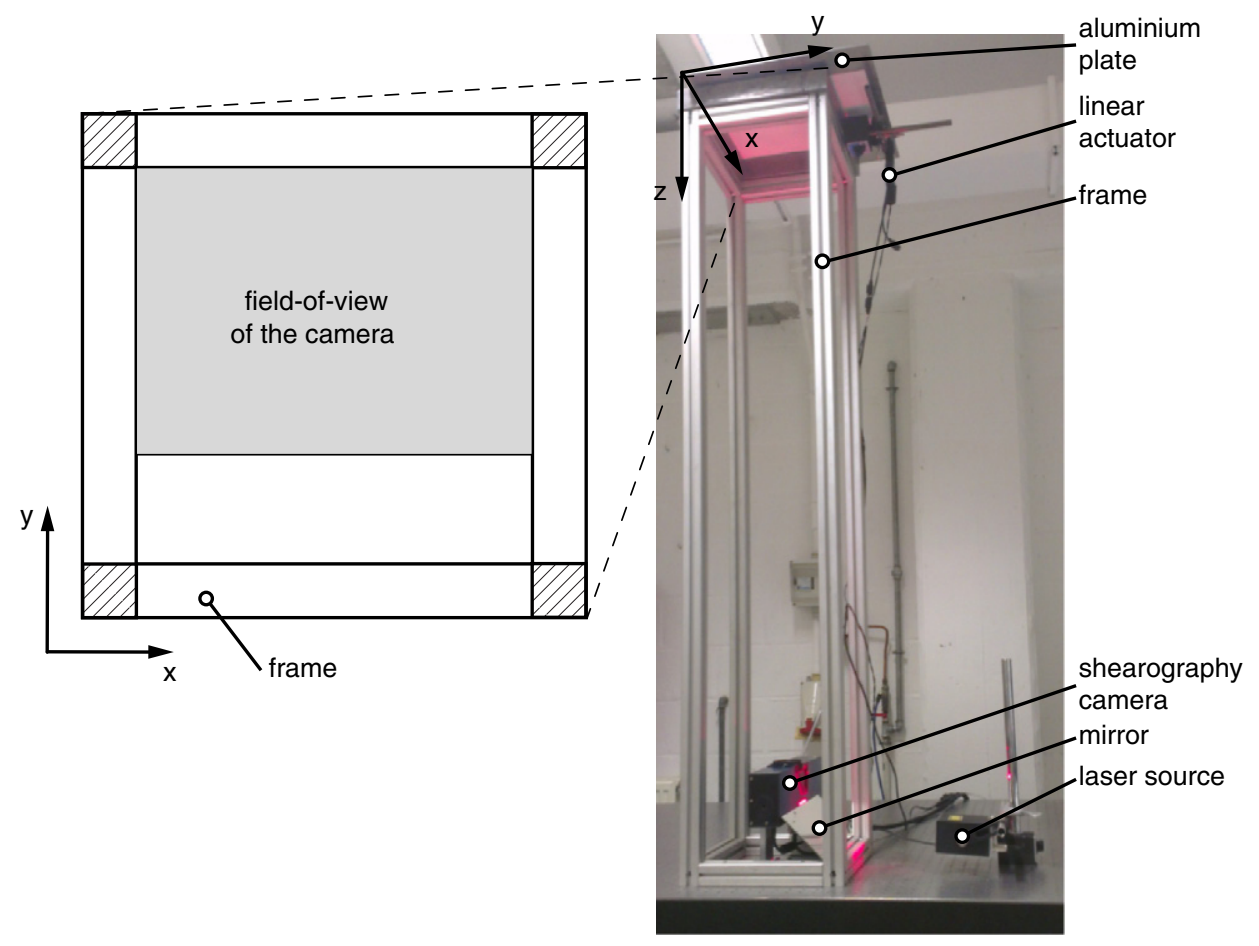

Figure 6. The experiments are done on a vertical setup where the shearography camera and the laser are placed below the plate. The field of view of the camera is illustrated on the left. The rigid-body rotations of the plate are performed by a rigid-body rotation mechanism, shown in more detail in figure 5.

mechanically. The phase-shifting algorithm used is the Carré algorithm [22, 23]:

$$
\tan ^{2} \Phi=\frac{\left.\left[3\left(I_{2}-I_{3}\right)-\left(I_{1}-I_{4}\right)\right]\left[\left(I_{2}-I_{3}\right)\right]+\left(I_{1}-I_{4}\right)\right]}{\left[\left(I_{2}+I_{3}\right)-\left(I_{1}-I_{4}\right)\right]^{2}}
$$

where a phase $\Phi$ in a point is calculated from the consecutively measured intensities $I_{1} \ldots I_{4}$. The intensities $I_{1} \ldots I_{4}$ feature phase-shifts, introduced by the phase-shifting mirror, shown in figure 2. In the Carré algorithm, the size of the phase-shifts can be arbitrarily chosen, as long as they are equally spaced.

The plate is rested on two ball rollers and a point which can be displaced by a linear actuator, as shown in figure 5 . The linear displacement of the actuator $t$ is acting on a point at a distance $l$ from the rotation axis, rotating the plate by an angle $\tan (t / l)$. Depending on whether the ball rollers are positioned along the $x$ - or the $y$-axis of the setup, the angle of the rotation will be $\theta_{x}$ or $\theta_{y}$. To induce the displacement $t$, the micro linear actuator Zaber T-NA08A25 is used. The actuator moves in step sizes of $t_{\text {step }}=47.6 \mathrm{~nm}$ and the displacement can only be in multiples of a step as $t=n t_{\text {step }}$, where $n$ is an integer number. The actuator is calibrated, with the standard deviation of the displacement $\sigma_{t}=0.4 \mu \mathrm{m}$, assuming a Gaussian distribution of the uncertainty.

If the laser were oriented vertically, it would warm the air just above it and the rising warm air would create heat refractions visible in the shearography images. Thus, the laser is turned horizontally and the plate is illuminated by laser light reflected over a mirror, as seen in figure 6 .

The average shearing distances $\overline{\delta_{x}}$ and $\overline{\delta_{y}}$ are directly measured. The local variability of the shearing distance in the image is implicit in the calibration maps $\alpha_{C x}$ and $\alpha_{C y}$. The average

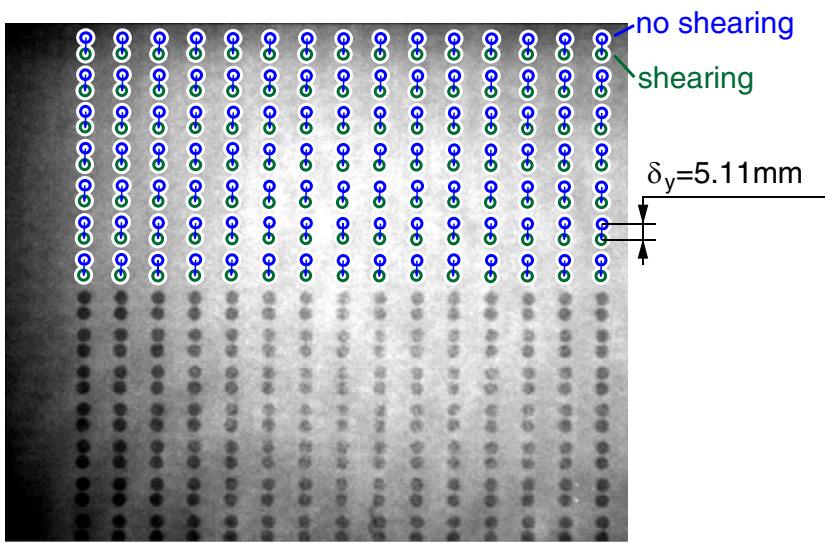

Figure 7. A dot pattern is placed instead of the specimen and from the shearing of the dots the average shearing distance is computed. Dots are automatically recognized and pairs of dots (one with no shearing and one with shearing) are matched. The upper half of the picture is showing the matched dots overlay, while the bottom half is showing only the unaltered image from the shearography camera.

shearing distances $\overline{\delta_{x}}$ and $\overline{\delta_{y}}$ are measured by replacing the measured surface by a flat plate with a $15 \times 14$ grid pattern of dots printed on it. The shearography camera doubles the image, overlaying the sheared and non-sheared images over each other. The $x$ and $y$ components of the separation between each sheared and non-sheared copy of a dot are the shearing distances $\delta_{x}$ and $\delta_{y}$. The analysis is automated, as the dots are easily recognized by a computer algorithm. In figure 7 the result of such an analysis is shown. As there are many dots over the field-of-view of the camera, the average shearing distance, as well as the standard deviation of the calculation, 
Table 1. Positions of the laser and the camera relative to the observed surface, for a typical configuration.

\begin{tabular}{lllr}
\hline & $x / \mathrm{mm}$ & $y / \mathrm{mm}$ & $z / \mathrm{mm}$ \\
\hline Camera detector (D) & 0 & 0 & 1444 \\
Laser source (S) & 0 & -65 & 1654 \\
Observed surface & {$[-130,130]$} & {$[-97,97]$} & 0 \\
\hline
\end{tabular}

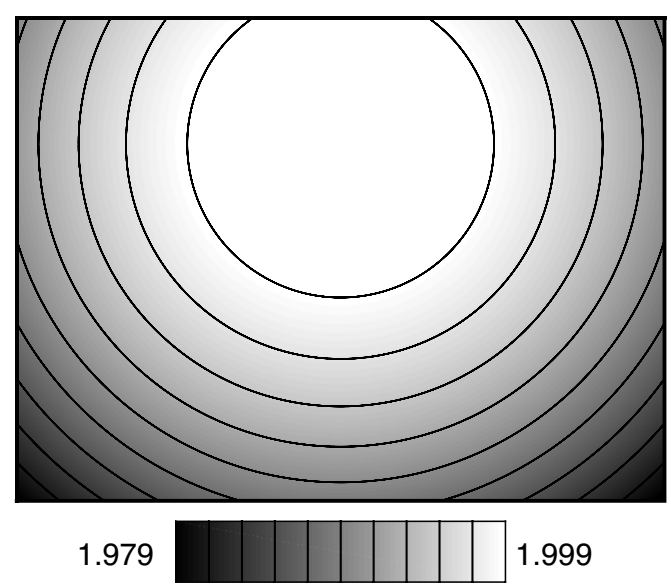

Figure 8. The local sensitivity map $k_{z}$ for a typical configuration for which average value $\overline{k_{z}}=1.993$.

can be computed with sufficient accuracy. As an example, in figure $7 \overline{\delta_{y}}=5.11 \mathrm{~mm}$ with standard deviation on the result $\sigma_{\delta y}=0.09 \mathrm{~mm}$ is obtained. In figure 7 the shearing in the $x$-direction $\overline{\delta_{x}}=0.20 \mathrm{~mm}$ is unintended. As is shown in equations (14) and (15), for the measurements in this section, the shearing in the perpendicular direction is not important as the gradient of displacement exists only in the dominant direction. More general examples with displacement gradients in both directions are considered in the next section.

Equations (17) show that also the out-of-plane sensitivity $k_{z}$ is needed for the calculation of the calibration maps $\alpha_{C x}$ and $\alpha_{C y}$. The sensitivity can be computed for each point in the field-of-view. For a typical experiment, the positions of the laser, of the camera and of the observed surface are given in table 1 . The field of $k_{z}(x, y)$ is calculated and shown in figure 8 . The value of the sensitivity $k_{z}$ only reaches 2 if the laser and the camera are located on the same line perpendicular to the measured surface, which is not practically possible as they would block each other's line of sight. In all practical cases $k_{z}<2$.

\subsection{Results}

Two sets of measurements need to be done for every combination of shearing angles and the aperture. One measurement, by observing the rotations around the $y$-axis for the angle $\theta_{y}$, is used to obtain the calibration map $\alpha_{C x}$ and the second, by observing the rotations around the $x$-axis by $\theta_{x}$, to obtain the $\alpha_{C y}$. The mechanism of two ball rollers and the actuator (figure 5) is rotated by $90^{\circ}$ around the $z$-axis between the measurements. The calibration maps $\alpha_{C x}$ and $\alpha_{C y}$ are not correlated. Both $\alpha_{C x}$ and $\alpha_{C y}$ are caused by the same internal optical distortions in the shearography camera but they are in the two independent directions.

The calibration maps are computed from the measured $\Delta$-images. However, wrapping can occur in the $\Delta$-images when higher rotation angles $\theta_{y}$ and $\theta_{x}$ are imposed. Standard unwrapping techniques cannot be used, as they rely on fringes to identify where jumps of $2 \pi$ occur. In the $\Delta$-images used here, the values across the images have relatively small variation and fringes rarely occur. Figure $9(a)$ shows that, as the rotation angle $\theta_{y}$ is increased, wrapping occurs. The wrapping shift is deduced from a known rotation angle $\theta_{y}$ and added to the $\Delta$-image. After addition of a wrapping shift (in figure $9(b)$ it is $\{0,1,2\} \cdot 2 \pi)$ the measured $\overline{\Delta_{x}}$ are on a single line. The co-linearity in figure $9(b)$ shows that the assumption in equation (16) is very well satisfied. The standard deviation of setting the angle $\theta_{y}$, calculated from the uncertainty of the linear actuator is $\sigma_{\theta}=1 \mu \mathrm{m}$, or $1.6 \%$ of the measurement range of the shearography configuration. This warrants the use of equations (17) as the calculated corrections from the calibration map are of the same order of magnitude. A leastsquare fit through all the points in figure $9(b)$ would actually yield a line $\theta_{y}=1.0016\left(\lambda \overline{\Delta_{x}}\right) /\left(2 \pi \overline{k_{z}} \overline{\delta_{x}}\right)$, but this will not be taken as a calibration coefficient as the discrepancy of $0.16 \%$ is below the uncertainty associated with both setting the angle $\theta_{y}$ and measuring the shearing distance $\overline{\delta_{x}}$. As the assumption in equation (16) is shown to be valid, equation (17) can be used.

Computing the calibration map $\alpha_{C x}$ from equation (17) yields a very noisy calibration map, shown in figure $10(a)$. The high-frequency noise, inherent in all shearography measurements, needs to be filtered out in order for the computed calibration map to be useful. The calibration map filtered once by a $21 \times 21$ median filter reveals the nature of the calibration map (figure 10(b)). However, even repeated filtering does not completely eliminate the noise. A large number of filtering repetitions can actually induce errors in itself, such as skewed values on the edges of the image and grainy appearance, seen in figure $10(c)$. Instead, the calibration maps are fitted by a parametric surface. Fitting a $5 \times 5$ and $6 \times 6$ order bi-polynomial surface gives good results, as shown in figures $10(d)$ and $(e)$. Order of $5 \times 5$ is chosen for subsequent measurements as a lower order polynomial gives more robust solutions and the differences between fits in figures $10(d)$ and $(e)$ are negligible.

From each rigid-body rotation measurement, a calibration map $\alpha_{C x}$ can be computed. Since there are transient errors due to the air-currents, four measurements for each rotation angle $\theta_{y}$ are done. For small rotation angles $\theta_{y}$, the influence of these transient errors is comparable to the influence of the optical distortions, thus the computation of the calibration maps has high uncertainty. For conciseness, these results are not shown. Generally, larger rotation angles are favourable for computing the calibration maps as the figures $10(c), 11(a)$ and $(b)$ show for $\theta_{y} \in\{126,378,630\} \mu \mathrm{rad}$. For large rotation angles the obtained calibration maps match very well and the influence of the transient errors is small.

Since multiple measurements of a calibration map are available, the influence of transient errors can be reduced 


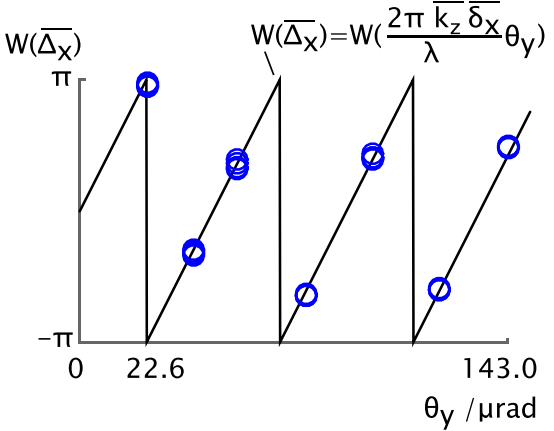

(a)

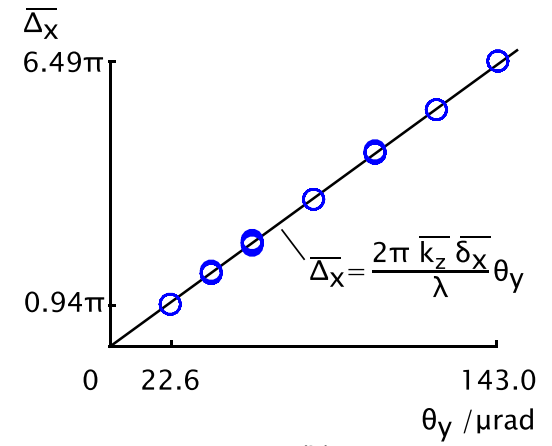

(b)

Figure 9. For the rotations around the $y$-axis, the average measured value fits the prescribed rotations very accurately. An unwrapping constant needs to be introduced to every $\Delta$-image to expand the values from the wrapped interval $[-\pi, \pi)(a)$ to the real interval $(b)$. The shearing distance is $\delta_{x}=7.39 \mathrm{~mm}$ and for each $\theta_{y}$ four measurements are done.

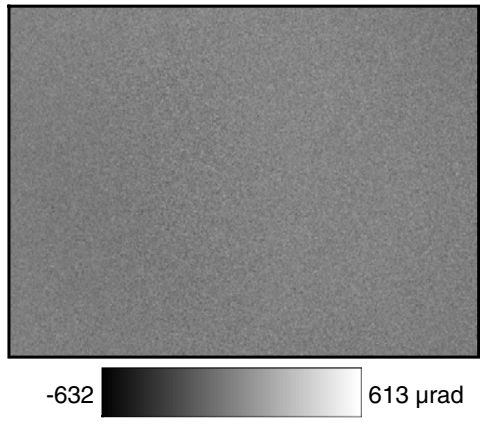

(a)

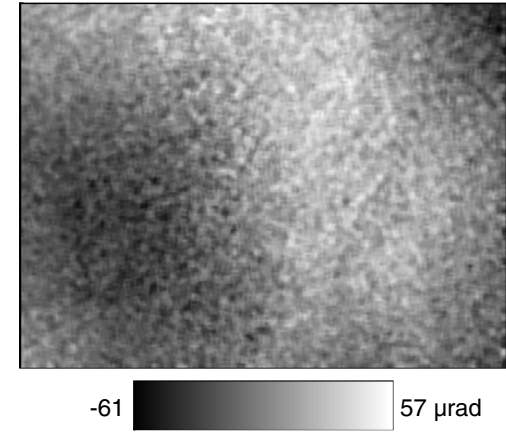

(b)

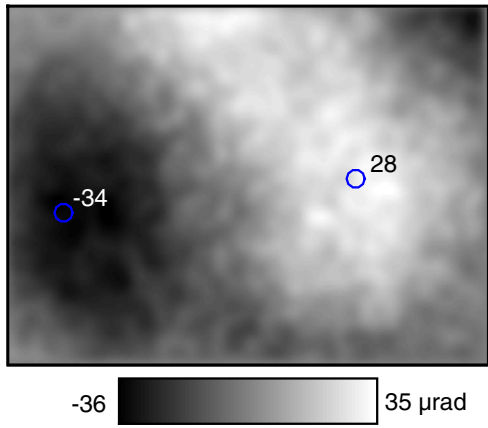

(c)

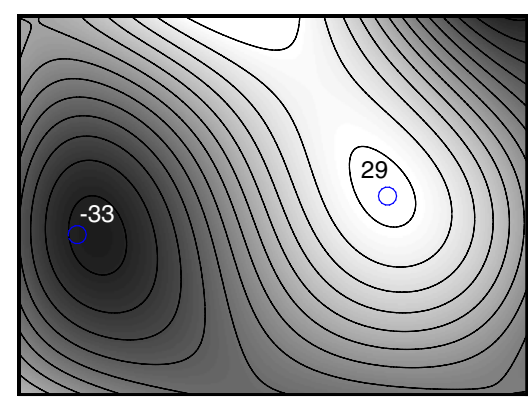

$-42$

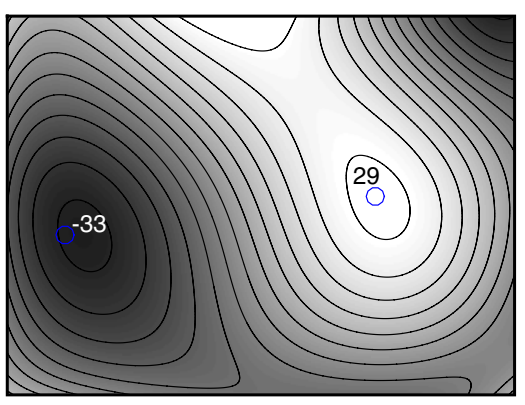

$-42$

(d)

(e)

Figure 10. Calibration map $\alpha_{C x}$ can be calculated from a single measurement of a rigid-body rotation. With no filtering the calibration map is overwhelmed by noise $(a)$. Filtering does not completely remove the influence of noise $(b)$ and repeated filtering is not a robust option as it can produce artefacts. A better option is fitting (without filtering) a parametric surface over the calibration map $(c, d)$. The $5 \times 5$ order bipolynomial surface fitting is chosen to be used in subsequent measurements. The calibration map is from a measurement with $\overline{\delta_{x}}=4.30 \mathrm{~mm}$ and $\theta_{y}=126 \mu \mathrm{rad}$.

by computing the mean calibration map from $n$ measurements as

$$
\alpha_{C x, \text { mean }}=\frac{1}{n} \sum^{n} \alpha_{C x, n}
$$

Furthermore, the uncertainty in the computation of the calibration map can be quantified. A standard deviation of a measurement is computed as

$$
\sigma=\sqrt{\frac{1}{n N_{x} N_{y}} \sum^{n} \sum^{N_{x}} \sum^{N_{y}}\left[\alpha_{C x, \text { mean }}(x, y)-\alpha_{C x, n}(x, y)\right]^{2}}
$$

where $N_{x}$ and $N_{y}$ are the image resolutions in the $x$ - and $y$-directions. The mean calibration map $\alpha_{C x \text {,mean }}$ shown in figure 11(c), computed from $n=12$ measurements, has a standard deviation of $\sigma=2.5 \mu \mathrm{rad}$, showing the measurements are consistent when varying the rotation angle $\theta_{y}$.

Figure 11 shows that there is no significant difference in the calibration maps when the angle $\theta_{y}$ is changed, as equation (17) predicts. Similarly, figure 12 shows that when the shearing angle $\overline{\alpha_{x}}$ is kept constant and the distance of the camera to the measured surface $d_{0}$ is varied the calibration maps are consistent. If the shearing angle $\overline{\alpha_{x}}$ is constant and the distance $d_{0}$ is changing, according to equation (12) $\overline{\delta_{x}}$ is changing. 


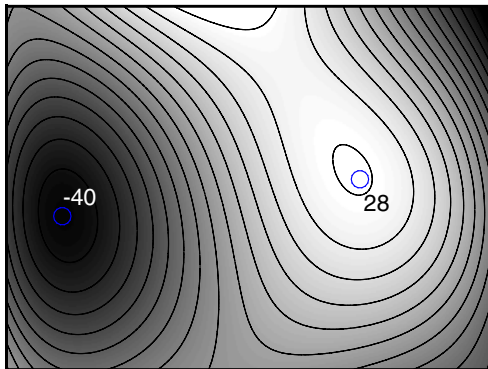

$-42$

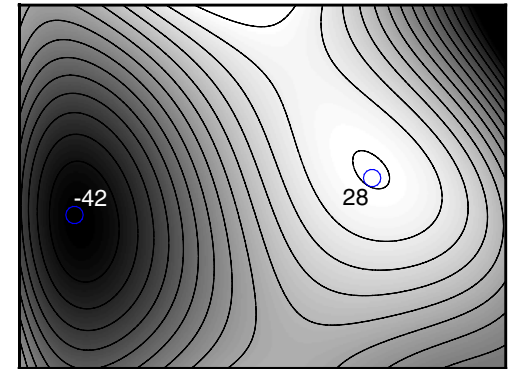

$-42$

(a)

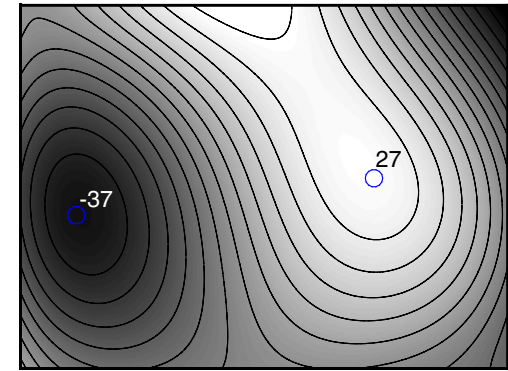

$-42$

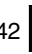

(b)

(c)

Figure 11. The calibration maps $\alpha_{C x}$ are consistent for varying angle of the rigid-body rotation $\theta_{y}$. When calibration maps $\alpha_{C x}$ from a single measurement are compared to the values averaged over more measurements, the standard deviation is $\sigma=2.5 \mu \mathrm{rad}$, showing good repeatability. The calibration maps are from a measurement with $\overline{\delta_{x}}=4.30 \mathrm{~mm}$. (a) $\theta_{y}=378 \mu \mathrm{rad},(b) \theta_{y}=630 \mu \mathrm{rad},(c)$ averaged $\alpha_{C x, \text { mean }}$, $n=12, \theta_{y}=\{126,378,630\} \mu \mathrm{rad}$.

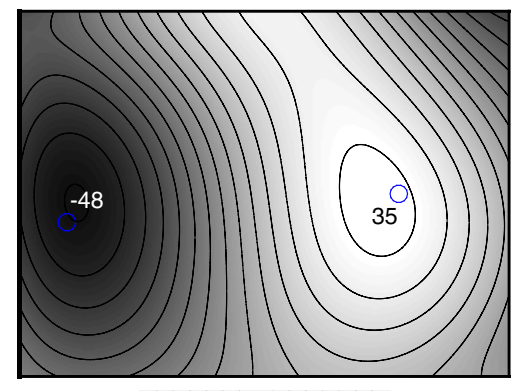

$-55$

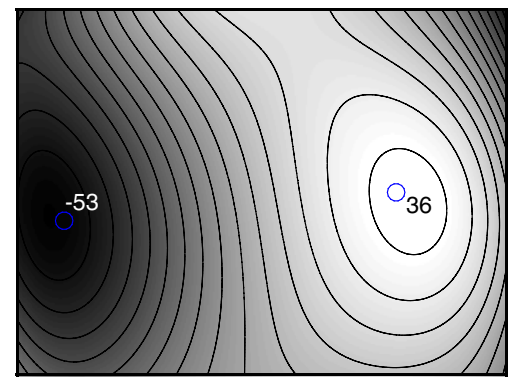

$-55$

(a)

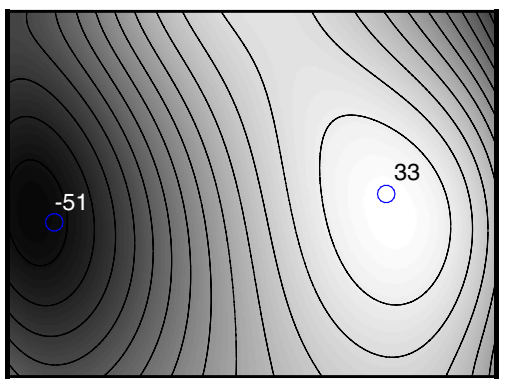

$-55$

(b)

(c)

Figure 12. The calibration maps $\alpha_{C x}$ are consistent when the distance $d_{0}$ between the camera and the object is changed and the shearing angle is constant $\overline{\alpha_{x}}=2475 \mu \mathrm{rad}$. As the distance $d_{0}$ is changing the shearing distance is changing also, according to equation (12). (a) $d_{0}=1.30 \mathrm{~m}, \overline{\delta_{x}}=6.30 \mathrm{~mm}$. (b) $d_{0}=1.01 \mathrm{~m}, \overline{\delta_{x}}=5.47 \mathrm{~mm}$. (c) $d_{0}=0.71 \mathrm{~m}, \overline{\delta_{x}}=3.95 \mathrm{~mm}$.

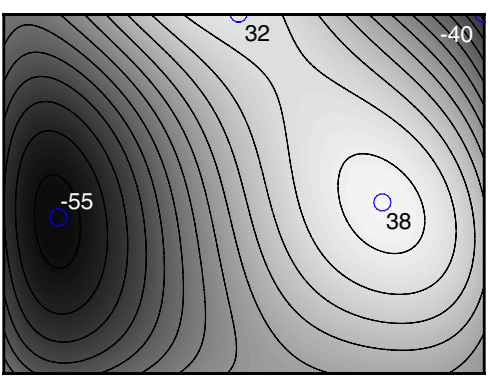

$-60$

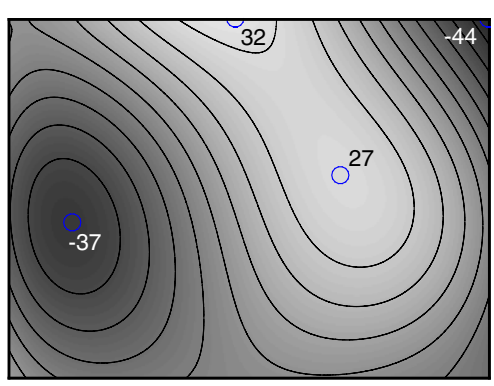

$-60$
$50 \mu \mathrm{rad}$

(a)

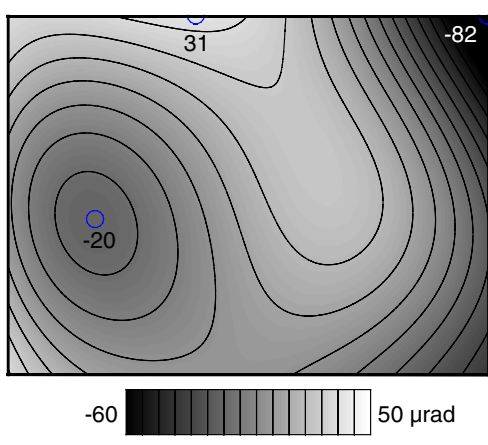

(c)

Figure 13. The calibration map $\overline{\alpha_{C x}}$ changes when the shearing angle $\alpha_{x}$ is changed, here represented by the average shearing distance $\overline{\delta_{x}}$. (a) $\overline{\delta_{x}}=7.3 \mathrm{~mm}$. (b) $\overline{\delta_{x}}=4.3 \mathrm{~mm}$. (c) $\overline{\delta_{x}}=-4.7 \mathrm{~mm}$.

The calibration maps $\alpha_{C x}$ are small compared to the average shearing angle $\overline{\alpha_{x}}$. For the case shown in figure 11(c), $\alpha_{C x}$ is in the interval $[-42,33] \mu \mathrm{rad}$ and $\overline{\alpha_{x}}=2110 \mu \mathrm{rad}$ (equivalent to the average and the shearing distance $\overline{\delta_{x}}=4.43 \mathrm{~mm}$ for this case). The correction of applying the calibration maps, by using equation (13), is thus also small, in the interval $[-2.0 \%$, $1.6 \%$ ] for this case.

The calibration map changes if the optical settings of the setup change. Figure 13 shows that the calibration map considerably changes as the shearing distance is varied. One calibration map is thus valid only for a certain value of the shearing distance. Figure 14 shows that changing the opening of the aperture affects the calibration map. The aperture controls the width of the light beam bundle that falls onto one pixel of the camera detector. A wide light beam bundle effectively 'averages' the optical distortions over a larger area of the lenses and mirrors. When the aperture opening is narrower the light bundle is thinner and the system is more susceptible 


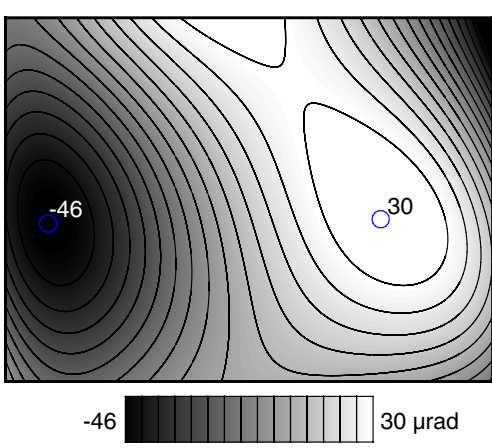

(a)

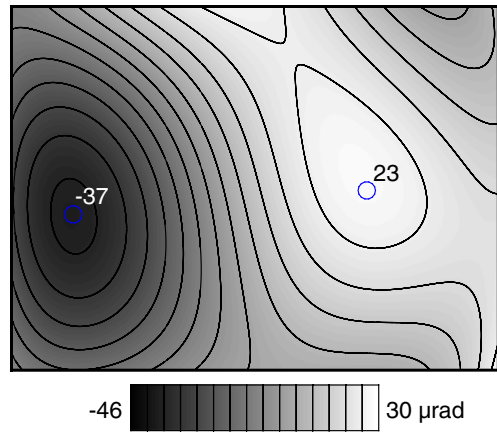

(b)

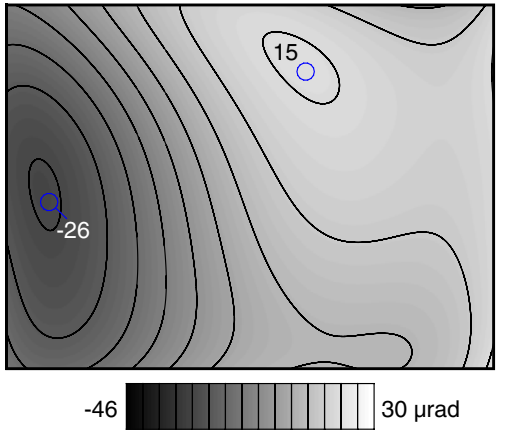

(c)

Figure 14. The calibration map $\alpha_{C x}$ is affected by the area of the aperture opening $S_{A}$. As the aperture opening is increased, the effects of the optical distortions are reduced. The aperture opening could not be practically measured in absolute terms, but it is possible to compare accurately two aperture openings. The opening area $S_{A}$ is directly proportional to the light admitted to the camera detector and thus the measured brightness in the image. The aperture openings $S_{A a}$ and $S_{A c}$ are the minimal and maximal practical aperture openings for the measurement. (a) Aperture $S_{A a},(b)$ aperture $S_{A b}=2.45 S_{A a},(c)$ aperture $S_{A c}=5.01 S_{A a}$.

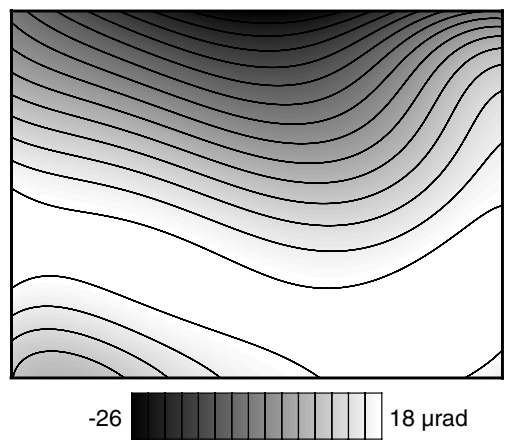

Figure 15. Calibration map $\alpha_{C y}$ averaged from all the measurements and fitted with a $5 \times 5$ order polynomial surface.

to the optical distortions. This effect is seen in figure 14. A narrow aperture increases the influence of the calibration map.

To ensure the experiments are not affected by the surface irregularities of the plate's surface, the experiments are repeated with the plate rotated around the $z$-axis and no change to the calibration maps is noted. A theoretical demonstration that the surface irregularities do not affect the calibration map measurements is provided in the appendix to this paper.

The calibration map $\alpha_{C y}$ is calculated for rigid-body rotation by $\theta_{x}$, from equation (17), analogously to the previous procedure for the $\alpha_{C x}$. A calibration map for a certain parameter set is shown in figure 15 .

With the calibration maps $\alpha_{C x}$ and $\alpha_{C y}$ known for a particular set of parameters the correction procedure can be performed for any experiments with those parameters. First the varying shearing distances $\delta_{x}$ and $\delta_{y}$ are computed from the measured average shearing distances $\overline{\delta_{x}}$ and $\overline{\delta_{y}}$ by applying the calibration map in equations (13). From two measurements of the same experiment with distinct shearing distances, by using equation (9), the exact deformation gradient can be computed. In the following section the correction method is tested on two cases: a rigid-body rotation of a plate with axis in an arbitrary direction and a clamped flat plate, centrally loaded by a weight, whereby a double-curved surface deformation is achieved.

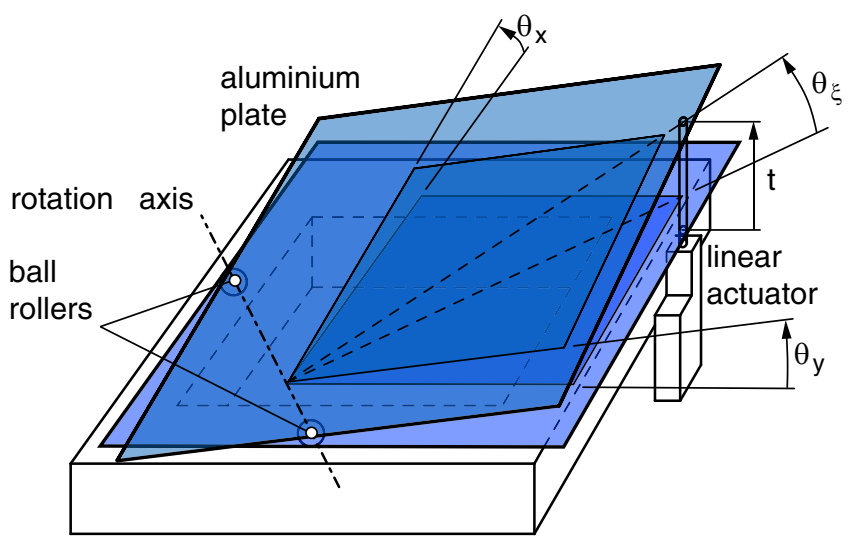

Figure 16. The schematic for the rigid-body rotation mechanism where gradient is present in both the $x$ - and the $y$-directions.

\section{Applying the correction procedure}

\subsection{Rotation of a flat plate around an arbitrary axis}

The measurement setup is the same as the one used in the previous section. The only difference is that the ball rollers are in different positions, as shown in figure 16. The displacement of the linear actuator $t$ is causing a gradient in both the $x$ - and the $y$-directions, characterised by the angles $\theta_{x}$ and $\theta_{y}$. Only one displacement of the linear actuator is used, $t=47.6 \mu \mathrm{m}$, causing the angles $\theta_{y}=-98.8 \mu \mathrm{rad}$ and $\theta_{x}=98.8 \mu \mathrm{rad}$. The shearing distances are such that for each measurement they are dominant in one direction. For the measurement 1 , they are dominant in the $x$-direction as $\overline{\delta_{x 1}}=6.45 \mathrm{~mm}$ and $\overline{\delta_{y 1}}=0.60 \mathrm{~mm}$. For the measurement 2, with shearing dominant in the $y$-direction, the shearing distances are $\overline{\delta_{x 2}}=0.12 \mathrm{~mm}$ and $\overline{\delta_{y 2}}=6.60 \mathrm{~mm}$.

If the correction and the decoupling of the gradients $\partial w / \partial x$ were not performed, the gradient field would be computed directly, using images $\Delta_{1}$ and $\Delta_{2}$ through equations (2). Figures 17(a) and (b) show the results for the uncorrected evaluation. The rigid-body rotation angles $\theta_{x}$ and $\theta_{y}$ are known, so the measured deformation gradient can be expressed in relative terms, compared to the angle value it should be. In figure 17(a), 


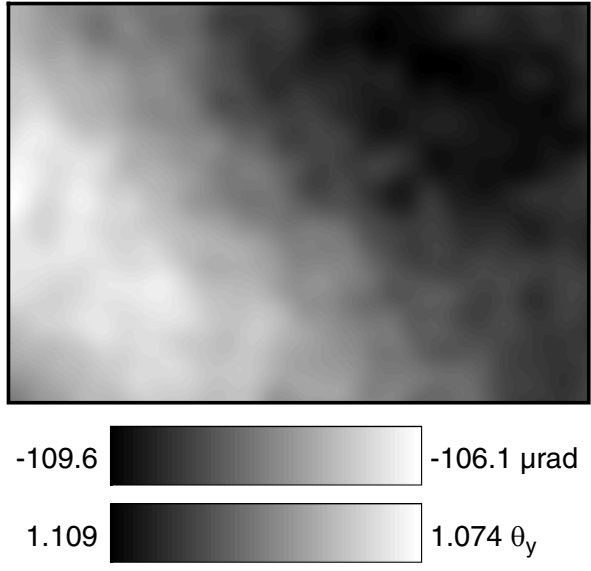

(a)
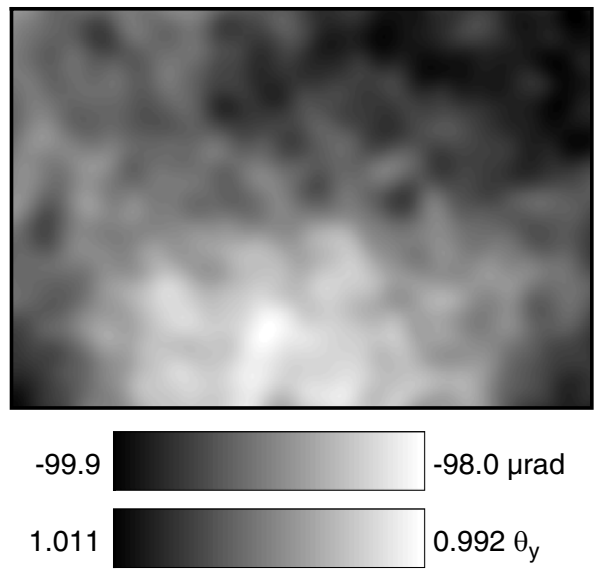

(c)
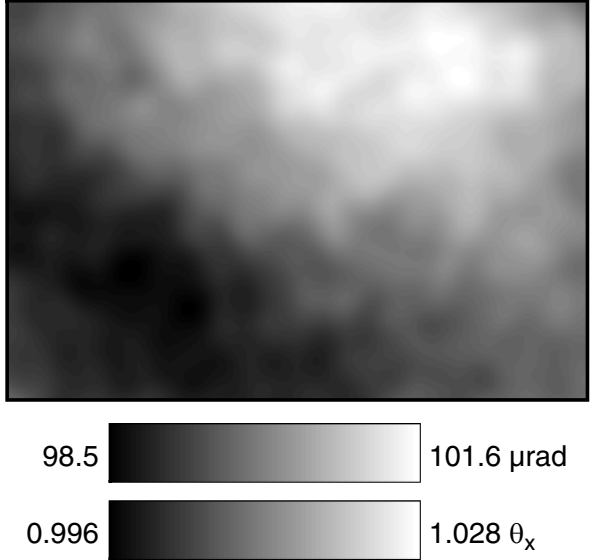

(b)

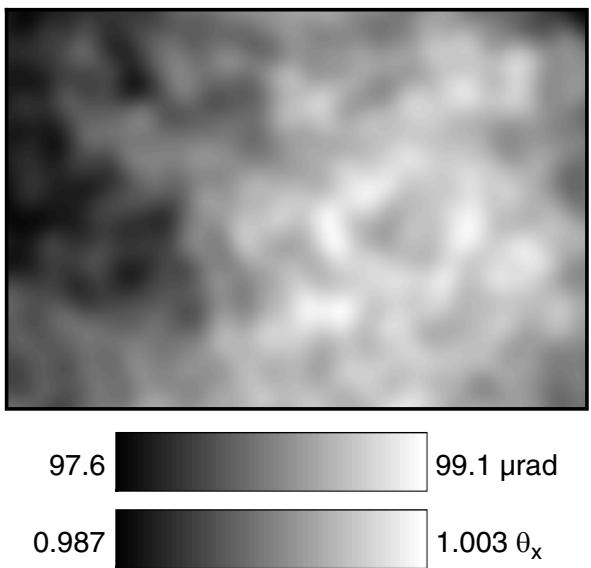

(d)

Figure 17. Without the correction procedure applied the displacement gradients $(a, b)$ are not uniform. The displacement gradients relative to the angles $\theta_{y}$ and $\theta_{x}$ are skewed from 1 . After the correction procedure is applied $(c, d)$ the resulting gradients are more uniform and the relative gradients are centred around 1 . Notice that since $\theta_{y}$ is negative, the relative scale's minimum and maximum values are switched in $(a)$ and $(c)$.

the measured gradient in the $x$-direction is overestimating the rotation, as the displacement gradients are in an interval of $[1.074,1.109]$ of the real $\theta_{y}$. The unwanted shearing $\overline{\delta_{y 1}}$ for the measurement 1 is relatively large, $\overline{\delta_{y 1}} \approx 10 \% \overline{\delta_{x 1}}$, which makes the uncorrected measurement shown in figure $17(a)$ have a particularly large relative error. The unwanted shearing is by definition a parameter which is not well controlled. The gradient calculated in the $y$-direction, shown in figure $17(b)$, is overestimating the rotation, with the displacement gradient in the range of $[0.996,1.028]$ of the real $\theta_{x}$.

When the calibration, correction and decoupling are considered, the measured gradients $\partial w / \partial x$ and $\partial w / \partial y$ in figures $17(c)$ and $(d)$ show an improved image. The relative ranges for both images are centred around 1 and the images themselves are more homogeneous, showing variation from the real value by about $\pm 1 \%$.

The images in figure 17 are filtered once by a median $21 \times 21$ filter (to remove the outliers) and 20 times by an averaging $21 \times 21$ filter. Using less filtering results in grainier images, thus features are harder to distinguish visually. However, the resulting ranges for images do not depend heavily on the amount of filtering. The use of a lot of filtering is justified to better distinguish the features in the images and

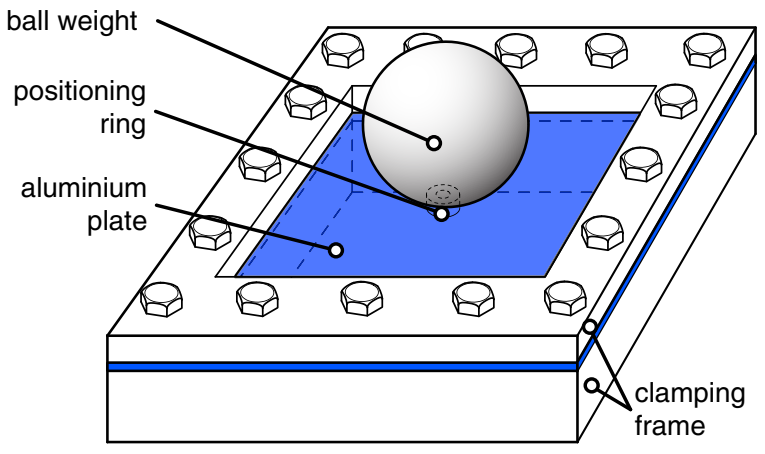

Figure 18. The schematic of the plate clamping and loading by the ball weight.

there is quantitatively little difference in using more or less filtering.

\subsection{A deformation of a centrally loaded plate}

In order to demonstrate the effect of the correction procedure on a realistic measurement case, an experiment with a double-curve surface deformed is devised. The experiment 


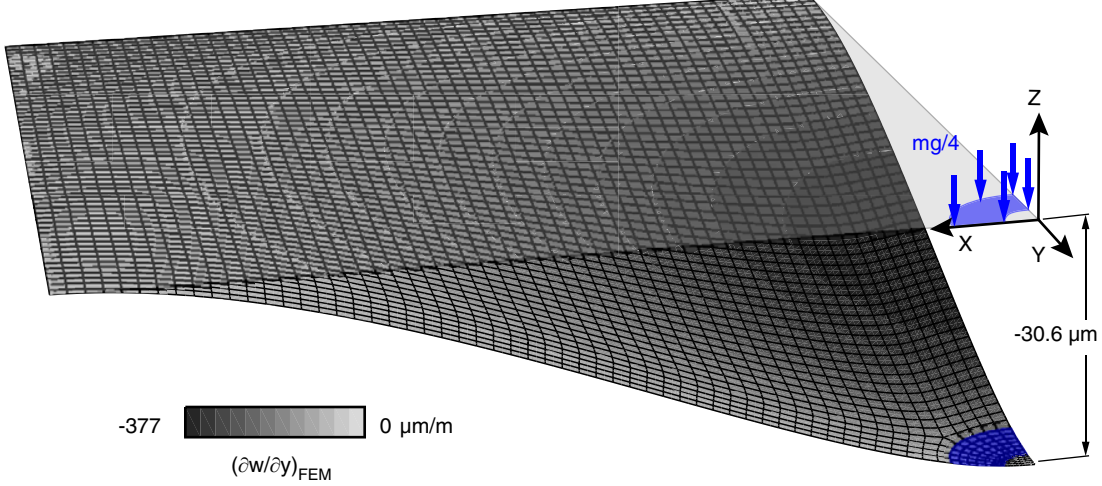

Figure 19. The finite element model of the quarter-plate. The deformation gradient $\partial w / \partial y$ is plotted on the deformed finite element mesh. The load in the model is applied as pressure over the ring area around the center, replicating the weight of the ball applied via the positioning ring.

consisting of a flat aluminium plate, statically deformed by a central load. The loaded plate with the clamping system is illustrated in figure 18. The loading conditions, the exact geometry of the plate and the boundary conditions of the experiment are matched as closely as possible by the FEM simulation.

The load asserted on the plate needs to be small, as shearography is intended for small-scale deformations. A steel ball weight is used to load the specimen as the weight can be accurately measured and converted into the load on the plate. The ball weight has a diameter of $D=100 \mathrm{~mm}$ and a mass of $m=4093.9 \mathrm{~g}$. To calculate the load from the weight, the gravitational acceleration $g=9.8152 \mathrm{~m} \mathrm{~s}^{-2}$ is estimated from the Earth gravitational model 2008 [39] at the location of the experiments.

As shown in figure 18, the ball weight is not placed on the plate directly. Instead, the ball weight is placed on a PVC ring glued to the middle of the aluminium plate, thus loading the plate indirectly. The purpose of the ring is to accurately position the weight at each loading and to evenly transmit the load onto a known area of the plate.

The aluminium plate has an area of $329.90 \times 330.31 \mathrm{~mm}^{2}$. From each side, $50 \mathrm{~mm}$ of the plate is clamped by a rigid steel frame. The rigidity of the frame is much higher than the rigidity of the plate. Fixed boundary conditions are imposed on the finite-element model to simulate the clamping.

The experimental setup is similar to the previous ones, with the rotation mechanism replaced by the clamping system and the weight. Again, the setup is oriented vertically-the camera is at the bottom, imaging the plate being vertically loaded by the weight.

5.2.1. The finite element model. The aluminium plate is modelled in the finite element analysis package ABAQUS, using shell finite elements. The plate and the loads are symmetrical around the $x z$ and the $y z$ planes, so only a quarter of the plate is modelled, employing symmetric boundary conditions. The mesh is composed of 3321 second order quadrilateral elements of type S8R. The ABAQUS element S8R is a reduced integration 8-node general shell element, capable of modelling thick shells. The material is modelled by an elastic linear isotropic model because the strains are small. A convergence analysis is performed to ensure the finite element mesh is fine enough to accurately model the deformation.

The in-plane components of the displacement are two orders of magnitude smaller than the out-of-plane displacement, maximum in-plane displacement being $0.7 \mu \mathrm{m}$, compared to the maximum out-of-plane displacement of $30.6 \mu \mathrm{m}$. Thus only the out-of-plane displacement $w$ is exported to MATLAB, where the values for the quarter plate are mirrored around the symmetry planes and numerically differentiated in the $x$ - or $y$-direction. The finite element based $\partial w / \partial x$ and $\partial w / \partial y$ are then directly compared to the experimentally obtained values. The finite-element mesh is shown in figure 19.

\subsubsection{Measuring the exact plate properties for the finite-element} model. The deformation of the plate needs to be very accurately matched by the finite element model. To ensure that, the finite element model must have the correct geometric and material properties. The thickness $t$ and the stiffness modulus $E$ of the plate are critical in the bending behaviour of the plate, so particular attention is given to accurately measure them.

The plate's surface is machined to have an even thickness so that the finite-element model corresponds well with the real plate. Using a mechanical dial gauge, the thickness over the surface of the plate is measured to be $t=3.90 \pm 0.01 \mathrm{~mm}$, showing acceptable variance. By measuring the mass of the plate $\left(m_{\mathrm{P}}=1130.2 \mathrm{~g}\right)$, the surface area $\left(329.90 \times 330.31 \mathrm{~mm}^{2}\right)$ and the measured density of the material $\left(\rho_{\mathrm{AL}}=2.725 \mathrm{~g} \mathrm{~cm}^{-3}\right)$, the average thickness can be more precisely calculated to $t=3.898 \mathrm{~mm}$.

The value of the stiffness modulus is only approximately known for the type of aluminium $E=68 \mathrm{GPa}$. The vibration behaviour of the plate is used to identify the stiffness. The plate, while clamped, is excited by tapping it with a hammer. The resulting vibrations are recorded using an accelerometer. The resonant frequencies are extracted from the measured accelerometer response and used to tune the stiffness modulus $E$ in the finite element model.

The resonant modes that were considered for measurement are the breathing mode $\left(f_{1}\right.$ mode) and the saddle mode $\left(f_{3}\right.$ mode) of the plate. These modes are used because they 


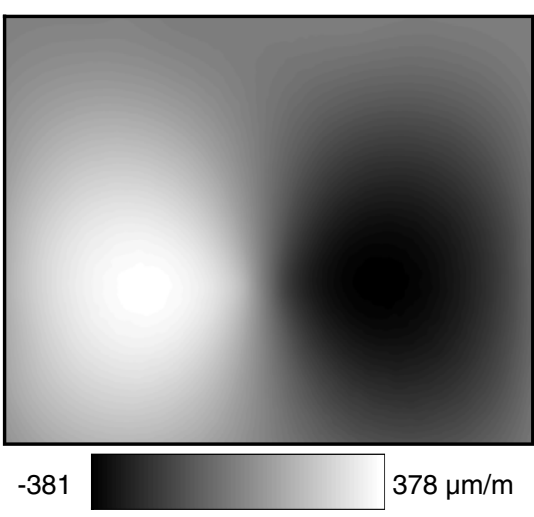

(a)

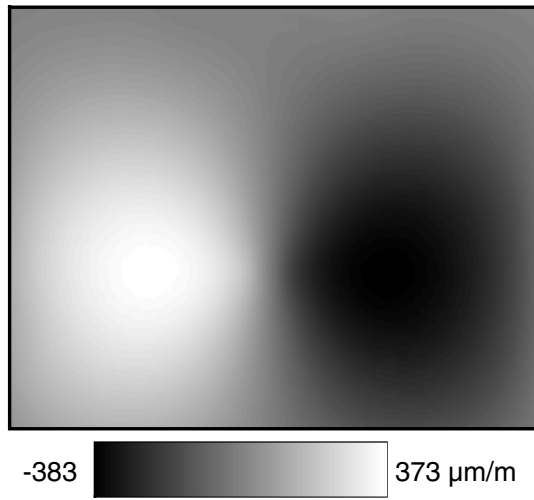

(c)

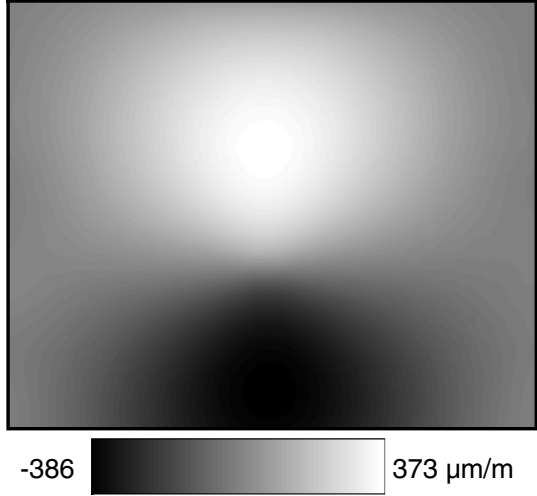

(b)

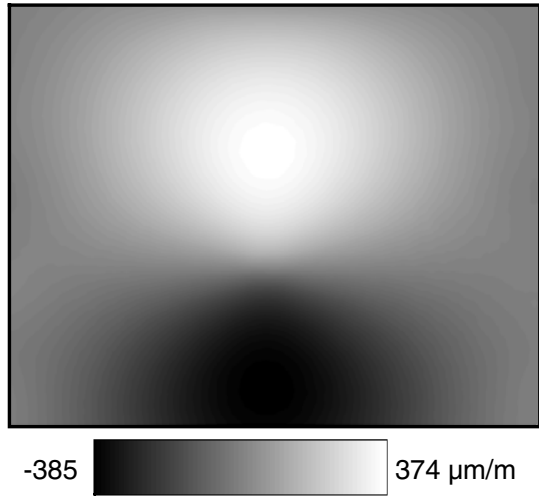

(d)

Figure 20. The deformation of the plate by the weight-without calibration and gradient decoupling $(a, b)$ and with $(c, d)$.

produced the clearest signal and they are separated enough from the other resonant frequencies as to not induce modemixing. Their measured values are $f_{1}=610.4 \mathrm{~Hz}$ (with standard deviation $\sigma_{f 1}=2.78 \mathrm{~Hz}$ from 13 repeated experiments) and $f_{3}=2275.1 \mathrm{~Hz}$ (with $\sigma_{f 3}=6.50 \mathrm{~Hz}$ from 14 repeated experiments). The stiffness modulus in the finite element model was tuned to the value of $E=68.93 \mathrm{GPa}$, producing the resonant frequencies $f_{1 \mathrm{FEM}}=611.65 \mathrm{~Hz}(+0.20 \%$ difference to the measurement) and $f_{3 \mathrm{FEM}}=2270.5 \mathrm{~Hz}$ $(-0.20 \%$ difference to the measurement). The Poisson's ratio is not expected to vary and it does not influence the frequency response significantly so it is not included in the tuning procedure. Instead, a typical Poisson's ratio for aluminium $\nu=0.33$ is used.

Using resonant frequencies has several advantages over the traditional way to obtain the stiffness modulus, measuring the stress-strain curve with a tensile test. The plate's modal shapes exhibit bending stresses, similar to the loading that will be used for the shearography measurements. The clamping is very hard to characterize and model well in FEM. Tuning the stiffness modulus $E$ to fit the resonant frequencies of a clamped plate also takes the clamping into account. Although the clamping is modelled as perfect in FEM, the stiffness modulus $E$ will account for the slight elasticity of the clamping. Measuring the frequencies is also simple, with each measurement lasting only several seconds and it produces accurate results. The procedure for measuring the resonant frequencies is the underlying principle for the
Resonalyser method [40], which can characterize isotropic and orthotropic material.

\subsubsection{Comparison of the measured results to the finite-element} model. The plate deformation is measured by shearography. An identical experiment is repeated 12 times with the shearing distance dominant in the $x$-direction and 12 times with the shearing distance dominant in the $y$-direction. The results with the same shearing distances were averaged, to remove the possible transient errors due to the air-currents and to increase the signal-to-noise ratio of the measurement. The shearing distances are measured to be $\overline{\delta_{1 x}}=6.1 \mathrm{~mm}$ and $\overline{\delta_{1 y}}=0.3 \mathrm{~mm}$, and $\overline{\delta_{2 x}}=0.2 \mathrm{~mm}$ and $\overline{\delta_{2 y}}=5.8 \mathrm{~mm}$ for the two sets of measurements with different shearing distances.

The $\Delta$-images with no corrections, using the simplified shearography equations (2), produce the gradients shown in figures $20(a)$ and $(b)$. The resulting gradients with the correction procedure are shown in figures $20(c)$ and $(d)$. Qualitatively, there are only slight differences.

A better way to compare the two maps is by looking at the residuals between the finite element analysis and the shearography measurements. The residuals are used as

$$
\begin{aligned}
& R_{D x}=\frac{\Delta_{1} \lambda /\left(4 \pi \overline{\delta_{1 x}}\right)-(\partial w / \partial x)_{\mathrm{FEM}}}{\operatorname{mean}|\partial w / \partial x|_{\mathrm{FEM}}} \\
& R_{D y}=\frac{\Delta_{2} \lambda /\left(4 \pi \overline{\delta_{2 y}}\right)-(\partial w / \partial y)_{\mathrm{FEM}}}{\operatorname{mean}|\partial w / \partial y|_{\mathrm{FEM}}}
\end{aligned}
$$




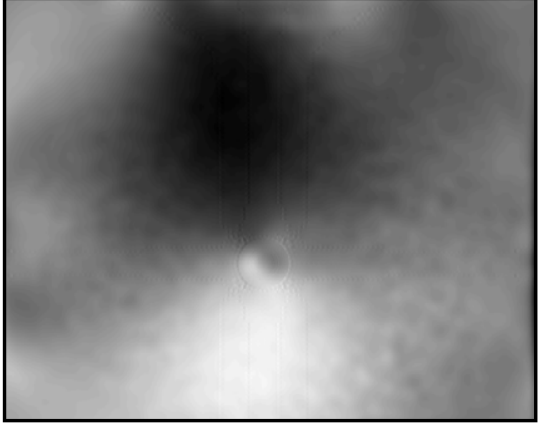

$-0.132$

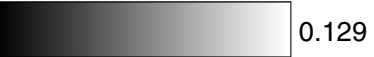

(a)

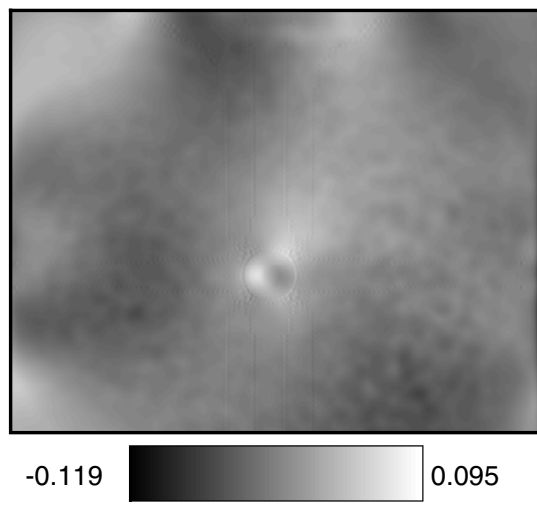

(c)

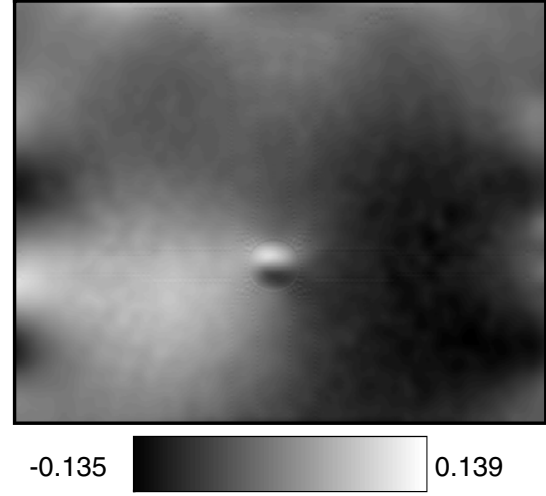

(b)

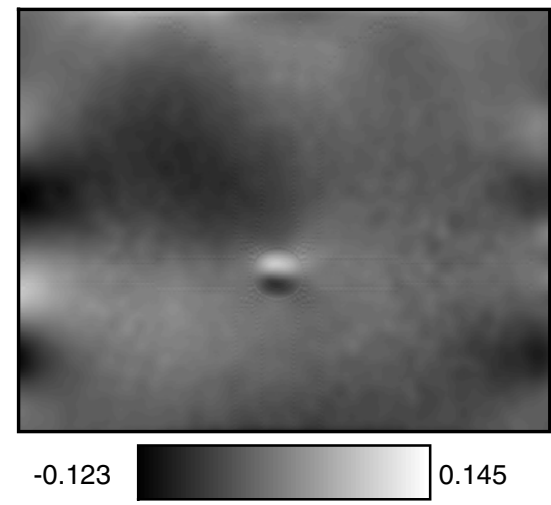

(d)

Figure 21. The residual comparing the finite element model analysis to the direct shearography measurements $(a, b)$ and to the corrected gradients $(c, d)$. The boundary conditions at the edges of images and the loading condition in the center clearly show discrepancy in the finite element model. Still, the corrected deformation gradient matches the finite element model better.

for the direct, uncorrected shearography measurements. For the shearography measurements after the correction procedure the residuals are computed as

$$
\begin{aligned}
R_{C x} & =\frac{\partial w / \partial x-(\partial w / \partial x)_{\mathrm{FEM}}}{\operatorname{mean}|\partial w / \partial x|_{\mathrm{FEM}}} \\
R_{C y} & =\frac{\partial w / \partial y-(\partial w / \partial y)_{\mathrm{FEM}}}{\operatorname{mean}|\partial w / \partial y|_{\mathrm{FEM}}}
\end{aligned}
$$

The residuals of the direct measurements with no corrections $R_{D x}$ and $R_{D y}$, shown in figures $21(a)$ and $(b)$, show the discrepancy between the finite elements model and the shearography measurements in the interval of $[-13 \%, 13 \%]$. This is normal, as the finite-element model does not capture all the subtleties of a physical experiment. However, there are distinct gradients over the image which are not necessarily a result of the finite element model limitations. The residuals of the corrected gradients show a more homogeneous image, with discrepancies in a slightly tighter interval, shown in figures $21(c)$ and $(d)$. The discrepancies are, however, located in the areas where the finite element model would be inconsistent with the physical reality of the experiment, in the area where the loading is applied in the FEM and near the clamping. The clamping is tightened by screws, which makes the clamping uneven over the edges, clearly seen in figures $21(c)$ and $(d)$. The finite element model does not take the uneven boundary conditions into account.
In figure 22 the same residuals from figure 21 are shown, but by excluding the regions near the boundary conditions and where the load is applied, thus making for a more relevant comparison between the corrected and the uncorrected shearography measurements. Here the effectiveness of the correction procedure can be shown quantitatively. Before the correction procedure the discrepancy between the finite element model and the shearography is in the range of $[-13 \%, 13 \%]$, like in the previous figures. The discrepancies after the correction procedure are reduced to a range of $[-8 \%, 6 \%]$.

Because of the unavoidable experimental conditions, such as the inhomogeneities of the deformed material, specimen manufacture tolerances, imperfect boundary condition and the limitations of the finite-element model, the fit between the finite-element analysis and the experiment is not perfect. After the correction, higher errors are present as compared to the first test case. Still, the comparison in figure 22 clearly shows an improved match between the experiment and the finite-element analysis after the correction procedure is applied.

\section{Conclusions}

This paper shows that the optical distortions in the Michelson interferometer lead to the local variation of the shearing values, which influences the results of shearography measurements. 


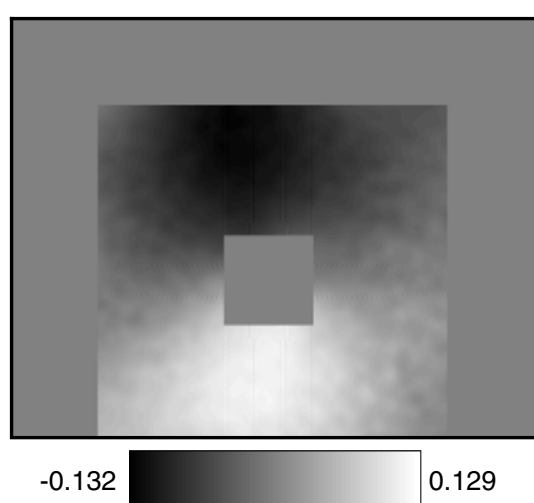

(a)

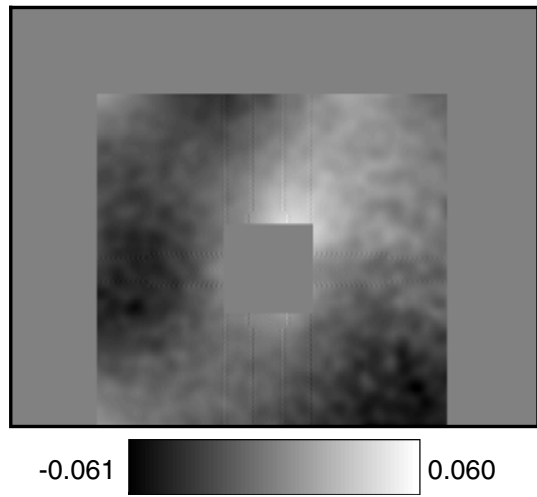

(c)

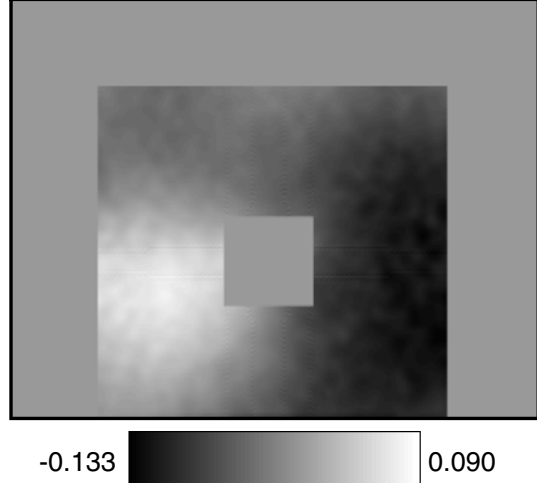

(b)

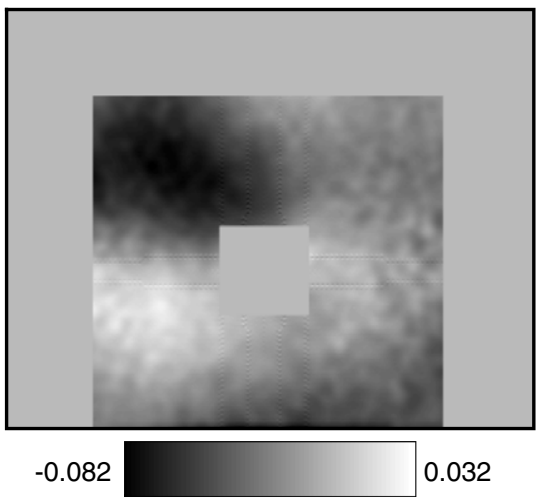

(d)

Figure 22. The residual comparing the final element model analysis to the direct shearography measurements $(a, b)$ and to the corrected gradients $(c, d)$, without taking into account the regions near the boundary conditions and the load. The discrepancies after the correction are in a narrower range of values, $[-8 \%, 6 \%]$, compared to the $[-13 \%, 13 \%]$ before the corrections.

The optical distortions depend on the angles of the shearing mirror $\alpha_{x}$ and $\alpha_{y}$ and the aperture opening $S_{A}$.

In this paper the procedure to calibrate and correct for systematic errors in shearography is developed. The calibration procedure is based on measuring the rigid-body rotations of a flat plate. By definition, the rigid-body rotations produce a constant displacement gradient, but the shearography images of the rigid-body rotation show slight variations in the measured displacement gradient field. From these variations and the known rotation angles, the calibration maps $\alpha_{C x}$ and $\alpha_{C y}$ are derived. The calibration maps $\alpha_{C x}$ and $\alpha_{C y}$ are 2-dimensional fields that are added to the shearing angles $\alpha_{x}$ and $\alpha_{y}$ to model the optical distortions in the Michelson interferometer. The proposed calibration needs to be performed for every combination of the shearing angles and aperture openings that will be used. The obtained calibration maps are used then in the correction procedure.

The shearography equations commonly contain one of the two possible shearing distances, $\delta_{x}$ or $\delta_{y}$. In the practical use of shearography, one of the shearing distances is dominant, but the other, though small, should not be neglected. In this paper the shearography equation with the influence of both shearing distances is derived, showing the gradient in the perpendicular direction is also coupled in the resulting shearography measurement. A part of the correction procedure proposed here, the gradient decoupling, is used to separate the measured gradients into gradients in the $x$ - and the $y$-directions only. For the gradient decoupling, pairs of shearography measurements of the repeated experiment are used, with different shearing distances $\delta_{x}$ and $\delta_{y}$.

Finally, the proposed process involving the calibration maps and the gradient decoupling is tested on a flat plate rotated around an arbitrary axis and a deformation of a centrally loaded plate. In the latter, a ball weight is used to deform a flat clamped plate. The experiments with and without using the proposed calibration and correction procedure are compared to each other and to the results from a finite-element simulation. The shearography measurements match the finite-element simulation well. The areas with the highest discrepancies are near the boundary conditions and the applied load, the areas where the finite element simulation is not expected to match the physical reality of the experiment exactly. The displacement gradients away from the boundary conditions and the load show a difference for the cases with and without the correction procedure applied. The direct shearography measurements, without the proposed correction procedures, show discrepancies in the interval [13\%, 13\%], while after corrections the discrepancies are reduced to the interval $[-8 \%, 6 \%]$.

The proposed procedure is not drastically changing the shearography images. The differences between the directly computed shearography measurements without the correction and the obtained gradients after the correction procedure is applied are 
up to about $5 \%$ for a normal case. However, when high accuracy measurements are required, these differences can have a meaningful influence on the results. The quantitative measurements with shearography can be done without calibration if an error of several percent is not an issue. All of the steps shown here, from isolating the external vibrations, correcting for the local shearing distance by using the calibration maps, correcting for the local sensitivity and decoupling the gradients, each contribute slightly, on the order of a few percent, to obtaining more accurate and reliable shearography measurements.

\section{Acknowledgments}

Part of this research has been sponsored by FWO, the Fund for scientific research of Flanders, Belgium.

\section{Appendix. Proof that the plate irregularities do not influence the calibration process}

There is a concern that the calibration process, using the rigidbody rotations, would be sensitive to the local irregularities of the flat plate used for the calibration. Thus a proof is provided here that the small irregularities in the plate do not pose a problem for the calibration procedure.

The schematic shown in figure A1 shows a cross-section of the plate used for the rigid-body rotation calibration in the $x-z$ plane. The rotation around the point $\mathrm{O}$ by an angle $\theta$ displaces the points $\mathrm{A}$ and $\mathrm{B}$ into $\mathrm{A}^{\prime}$ and $\mathrm{B}^{\prime}$ respectively. The point $\mathrm{B}$, however, is not in the plane of the plate, but is displaced in the $z$-direction by $z_{B}$. The point $\mathrm{B}$ is orthogonal to the point $\mathrm{B}_{0}$ on the $x-y$ plane.

The displacements in the $z$-direction of the points $\mathrm{A}$ and $\mathrm{B}_{0}$ on the $x-y$ plane are

$$
\begin{aligned}
w_{A} & =\sin \theta x_{A} \simeq \theta x_{A} \\
w_{B 0} & =\sin \theta x_{B} \simeq \theta x_{B}
\end{aligned}
$$

where the distances $x_{A}=\overline{\mathrm{OA}}=\overline{\mathrm{OA}}^{\prime}$ and $x_{B}=\overline{\mathrm{OB}}=\overline{\mathrm{OB}}^{\prime}$. The in-plane displacement of the point $\mathrm{A}$ can be neglected as $\left\{u_{A}, u_{B 0}\right\}=(1-\cos \theta)\left\{x_{A}, x_{B}\right\} \simeq 0$.

The approximation denoted by ' $\simeq$ ' means only the first term in the Taylor expansion of the sine and cosine function is taken into account. This is warranted as the rigid-body rotation angle $\theta$ is small. For the rigid-body rotation angles $\theta<200 \mu \mathrm{rad}$, as used in this paper, the error in neglecting all but the first term in the Taylor expansion of the sine and cosine functions is below $2 \times 10^{-8}$.

The distance $z_{B^{\prime}}$ after the rigid-body rotation is $z_{B^{\prime}}=\cos \theta z_{B}$ $\simeq z_{B}$ making the absolute displacement of the point $\mathrm{B}$

$$
\begin{aligned}
w_{B} & =w_{B 0}+z_{B^{\prime}}-z_{B}=\sin \theta x_{B}+\cos \theta z_{b}-z_{b} \\
& \simeq \theta x_{b}+z_{B}-z_{B}=\theta x_{B}
\end{aligned}
$$

The measured gradient of displacement between the points $\mathrm{A}$ and $\mathrm{B}$ can now be defined as

$$
\frac{\partial w_{A B}}{\partial x} \simeq \frac{w_{B}-w_{A}}{x_{B}-x_{A}}=\frac{\theta x_{B}-\theta x_{A}}{x_{B}-x_{A}}=\theta
$$

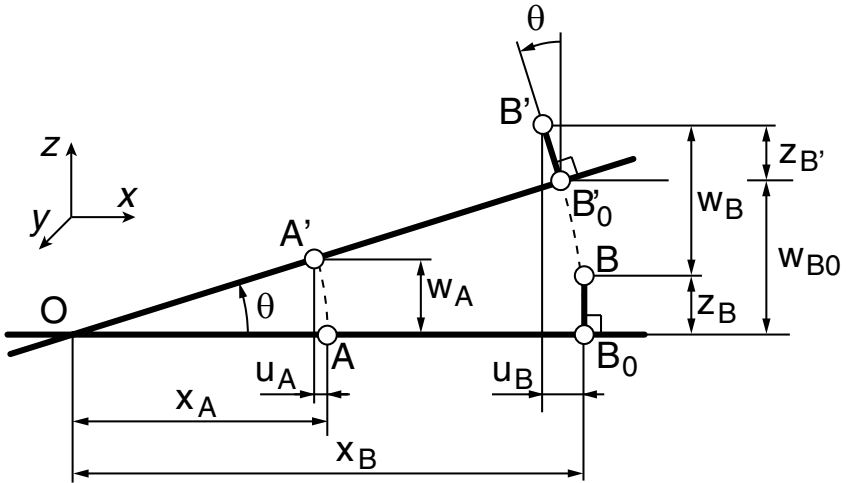

Figure A1. The schematic for the effect of the irregularities in the plate geometry.

showing that the measured gradient does not depend on the irregularities in the plate.

The in-plane displacement of the point B can be defined as

$$
u_{B}=(1-\cos \theta) x_{B}+\sin \theta z_{B} \simeq \theta z_{B}
$$

which means the in-plane displacement can become problematic if the irregularities in the plate are large. As $z_{B}$ is typically orders of magnitude smaller than $x_{B}$ so is $u_{B}$ smaller than $w_{B}$ so the in-plane displacement $u_{B}$ can be neglected. Additionally, the setup used is not sensitive to the in-plane displacements.

\section{References}

[1] Steinchen W and Yang L 2003 Digital Shearography: Theory and Application of Digital Speckle Pattern Shearing Interferometry (Bellingham, WA: SPIE Press)

[2] Francis D, Tatam R P and Groves R M 2010 Shearography technology and applications: a review Meas. Sci. Technol. 21102001

[3] Leendertz J A and Butters J N 1973 An image-shearing speckle-pattern interferometer for measuring bending moments J. Phys. E: Sci. Instrum. 6 1107-10

[4] Hung Y Y 1982 Shearography: a new optical method for strain measurement and nondestructive testing Opt. Eng. 21213391

[5] Hung Y 1999 Applications of digital shearography for testing of composite structures Composites B 30 765-73

[6] Mäckel P, Heyen H and Steinchen W 2007 Die Scherografie: Ein quantitatives Messverfahren zur Schwingungsmessung und zerstörungsfreien Prüfung Laser Technik J. 1 49-54

[7] Hung Y Y 1996 Shearography for non-destructive evaluation of composite structures Opt. Lasers Eng. 24 161-82

[8] Hung Y Y and Ho H P 2005 Shearography: an optical measurement technique and applications Mater. Sci. Eng. R 49 61-87

[9] De Angelis G, Meo M, Almond D P, Pickering S G and Angioni S L 2012 A new technique to detect defect size and depth in composite structures using digital shearography and unconstrained optimization NDT \& E Int. 45 91-6

[10] Groves R M, Chehura E, Li W, Staines S E, James S W and Tatam R P 2007 Surface strain measurement: a comparison of speckle shearing interferometry and optical fibre Bragg gratings with resistance foil strain gauges Meas. Sci. Technol. 18 1175-84

[11] Nelson D V 2010 Residual stress determination by hole drilling combined with optical methods Exp. Mech. 50 145-58 
[12] Groves R M, James S W and Tatam R P 2004 Shape and slope measurement by source displacement in shearography $O p t$. Lasers Eng. 41 621-34

[13] Zastavnik F, Pyl L, Gu J, Sol H, Kersemans M and Van Paepegem W 2014 Comparison of shearography to scanning laser vibrometry as methods for local stiffness identification of beams Strain 50 82-94

[14] Zastavnik F, Pyl L, Sol H, Kersemans M and Van Paepegem W 2014 Local stiffness identification of beams using shearography and inverse methods Advancement of Optical Methods in Experimental Mechanics (Conf. Proc. of the Society for Experimental Mechanics vol 3) ed H Jin et al (New York: Springer) pp 275-81

[15] Burguete R, Hack E, Patterson E, Siebert T and Whelan M 2010 Guidelines for the Calibration and Evaluation of Optical Systems for Strain Measurement, SPOTS (University of Sheffield)

[16] Hung Y Y and Liang CY 1979 Image-shearing camera for direct measurement of surface strains Appl. Opt. 18 1046-51

[17] Ghiglia D C and Pritt M D 1998 Two-Dimensional Phase Unwrapping: Theory, Algorithms and Software (New York: Wiley)

[18] Aebischer H A and Waldner S P 1999 A simple and effective method for filtering speckle-interferometric phase fringe patterns Opt. Commun. 162 205-10

[19] Fulton J P, Namkung M and Melvin L D 1993 Practical estimates of the errors associated with the governing shearography equation Rev. Prog. Quant. Nondestr. Eval. 12 427-34

[20] Steinchen W, Yang L X, Kupfer G, Mäckel P and Vössing F 1998 Strain analysis by means of digital shearography: potential, limitations and demonstration J. Strain Anal. Eng. Des. 33 171-82

[21] Zastavnik F, Pyl L, Gu J, Sol H, Kersemans M and Van Paepegem W 2014 Errors in shearography measurements due to the creep of the PZT shearing actuator: own typesetting Meas. Sci. Technol. 25085007

[22] Carré P 1966 Installation et utilisation du comparateur photoélectrique et interférentiel du Bureau International des Poids et Mesures Metrologia 2 13-23

[23] Qian K, Shu F and Wu X 2000 Determination of the best phase step of the Carré algorithm in phase shifting interferometry Meas. Sci. Technol. 11 1220-3

[24] Hariharan P, Oreb B F and Eiju T 1987 Digital phaseshifting interferometry: a simple error compensating phase calculation algorithm Appl. Opt. 26 2504-6

[25] Joenathan C 1994 Phase-measuring interferometry: new methods and error analysis Appl. Opt. 33 4147-55

[26] Hack E and Burke J 2011 Invited review article: measurement uncertainty of linear phase-stepping algorithms Rev. Sci. Instrum. 8206110
[27] Farrant D I and Petzing J N 2003 Sensitivity errors in interferometric deformation metrology Appl. Opt. $425634-41$

[28] Kästle R, Hack E and Sennhauser U 1999 Multiwavelength shearography for quantitative measurements of twodimensional strain distributions Appl. Opt. 38 96-100

[29] Waldner S P 2000 Quantitative strain analysis with image shearing speckle pattern interferometry (shearography) PhD Thesis Swiss Federal Institute of Technology, Zurich

[30] Francis D, James S W and Tatam R P 2007 Surface strain measurement using multi-component shearography with coherent fibre-optic imaging bundles Meas. Sci. Technol. 18 3583-91

[31] Goto D T and Groves R M 2010 A combined experiment with simulation approach to calibrated 3D strain measurement using shearography Proc. SPIE 7387 73871J

[32] Ng T W 1995 Shear measurement in digital speckle shearing interferometry using digital correlation Opt. Commun. $115241-4$

[33] Andersson A, Krishna N, Sjödahl M and Molin N E 2000 TV shearography: quantitative measurement of shearmagnitude fields by use of digital speckle photography Appl. Opt. $392565-8$

[34] Lee J-R, Yoon D-J, Kim J-S and Vautrin A 2008 Investigation of shear distance in Michelson interferometer-based shearography for mechanical characterization Meas. Sci. Technol. 19115303

[35] Aebischer H A and Rechsteiner P 1997 Theoretical prediction of the effect of shear distortion in the Michelson interferometer Pure Appl. Opt.: J. Eur. Opt. Soc. A 6 303-8

[36] Francis D, James S W and Tatam R P 2008 Surface strain measurement of rotating objects using pulsed laser shearography with coherent fibre-optic imaging bundles Meas. Sci. Technol. 19105301

[37] Hack E 2000 Rigid body effects in shearographic strain measurements Interferometry in Speckle Light ed P Jacquot and J-M Fournier (Berlin: Springer) pp 327-34

[38] Rosso V, Zhang L, Michel F, Renotte Y, Lion Y and Habraken A-M 2006 Out-of-plane displacement derivative measurement: comparison of results obtained by a shearographic interferometer using the separation of the polarization states and the finite element method Proc. SPIE $\mathbf{6 3 4 3} 634311$

[39] Pavlis N K, Holmes S A, Kenyon S C and Factor J K 2012 The development and evaluation of the Earth Gravitational Model 2008 J. Geophys. Res. 117 B04406

[40] Lauwagie T, Sol H, Roebben G, Heylen W and Shi Y 2002 Validation of the Resonalyser method: an inverse method for material identification Proc. ISMA 2002 (Leuven, Belgium, 16-18 September 2002) pp 687-94 